\title{
On Sub-Riemannian Geodesics in SE(3) Whose Spatial Projections do not Have Cusps
}

\author{
R. Duits ${ }^{1,2}$ - A. Ghosh ${ }^{3}$ T. C. J. Dela Haije ${ }^{1}$. \\ A. Mashtakov ${ }^{2}$
}

Received: 14 May 2013 / Revised: 19 April 2016/ Published online: 9 July 2016

(C) The Author(s) 2016. This article is published with open access at Springerlink.com

Abstract We consider the problem $\mathbf{P}_{\text {curve }}$ of minimizing $\int_{0}^{L} \sqrt{\xi^{2}+\kappa^{2}(s)}$ d $s$ for a curve $\mathbf{x}$ in $\mathbb{R}^{3}$ with fixed boundary points and directions. Here, the total length $L \geq 0$ is free, $s$ denotes the arclength parameter, $\kappa$ denotes the absolute curvature of $\mathbf{x}$, and $\xi>0$ is constant. We lift problem $\mathbf{P}_{\text {curve }}$ on $\mathbb{R}^{3}$ to a sub-Riemannian problem $\mathbf{P}_{\text {mec }}$ on $\mathrm{SE}(3) /(\{\mathbf{0}\} \times \operatorname{SO}(2))$. Here, for admissible boundary conditions, the spatial projections of sub-Riemannian geodesics do not exhibit cusps and they solve problem $\mathbf{P}_{\text {curve }}$. We apply the Pontryagin Maximum Principle (PMP) and prove Liouville integrability of the Hamiltonian system. We derive explicit analytic formulas for such sub-Riemannian geodesics, relying on the co-adjoint orbit structure, an underlying Cartan connection, and the matrix representation of SE(3) arising in the Cartan-matrix. These formulas allow us to extract geometrical properties of the sub-Riemannian geodesics with cuspless projection, such as planarity conditions, explicit bounds on their torsion, and their symmetries. Furthermore, they allow us to parameterize all admissible boundary conditions reachable by geodesics with cuspless spatial projection. Such projections lay in the upper half space. We prove this for most cases, and the rest is checked numerically. Finally, we employ the formulas to numerically solve the boundary value problem, and visualize the set of admissible boundary conditions.

Keywords Sub-Riemannian geometry $\cdot$ Special Euclidean motion group · Pontryagin Maximum Principle · Geodesics

R. Duits

r.duits@tue.nl

1 Department of Mathematics and Computer Science (CASA, Centre of Analysis, Scientific Computing and Applications), Eindhoven University of Technology, Eindhoven, The Netherlands

2 Department of Biomedical Engineering (Biomedical Image Analysis), Eindhoven University of Technology, Eindhoven, The Netherlands

3 Department of Mathematics (MTM, Mathematics and Applied Mathematics), Linköping University, Linköping, Sweden 
Mathematics Subject Classification (2010) 53C17 · 22E30 · 49J15

\section{Introduction}

In the space of smooth curves in $\mathbb{R}^{3}$, we define the energy functional

$$
\mathcal{E}(\mathbf{x}):=\int_{0}^{L} \sqrt{\xi^{2}+\kappa^{2}(s)} \mathrm{d} s, \quad \mathcal{E}: \mathcal{C}^{\infty}\left(\mathbb{R}, \mathbb{R}^{3}\right) \rightarrow \mathbb{R}^{+},
$$

with $L \in \mathbb{R}^{+}$being the length (free) of a curve $s \mapsto \mathbf{x}(s) \in \mathbb{R}^{3}$. Here, $\xi>0$ is a constant, $s$ denotes the arclength of the curve $\mathbf{x}$ and $\kappa:(0, L) \rightarrow \mathbb{R}^{+}$denotes the absolute curvature $\kappa(s)=\left\|\mathbf{x}^{\prime \prime}(s)\right\|$ of the curve $\mathbf{x}$ for all $s \in(0, L)$.

In this paper, we consider the problem $\mathbf{P}_{\text {curve }}$ of minimizing the functional $\mathcal{E}(\mathbf{x})$ among all smooth curves $s \mapsto \mathbf{x}(s)$ in $\mathbb{R}^{3}$, satisfying the boundary conditions (see Fig. 1)

$$
\mathbf{x}(0)=\mathbf{x}_{0}, \mathbf{x}(L)=\mathbf{x}_{1} \in \mathbb{R}^{3}, \quad \mathbf{x}^{\prime}(0)=\mathbf{n}_{0}, \mathbf{x}^{\prime}(L)=\mathbf{n}_{1} \in \mathrm{S}^{2} .
$$

Here, we parameterize $\mathbf{x}$ by spatial arclength, i.e., $\left\|\mathbf{x}^{\prime}(s)\right\|=1$, and via ordinary parallel transport on the tangent bundle $T\left(\mathbb{R}^{3}\right)$ the tangent vector $\mathbf{x}^{\prime}(s) \in T_{\mathbf{x}(s)}\left(\mathbb{R}^{3}\right)$ can be identified with a point $\mathbf{n}(s) \in S^{2}$.

The two-dimensional analog of this variational problem was studied as a possible model of the mechanism used by the visual cortex V1 of the human brain to reconstruct curves which are partially corrupted or hidden from observation. The two-dimensional model was initially due to Petitot (see [28, 29] and references therein). Subsequently, Citti and Sarti $[9,34]$ recognized the sub-Riemannian Euclidean motion group structure of the problem. In [5], the existence of minimizers was studied by Boscain, Charlot, and Rossi. It turned out that only for certain end conditions, the $2 \mathrm{D}$ problem $\mathbf{P}_{\text {curve }}$ is well-posed. Characterization of the set of end conditions for which $\mathbf{P}_{\text {curve }}$ is well-posed can be found in [11]. The more general 2D problem related to a mechanical problem was completely solved by Sachkov [25, 32, 33], who in particular derived explicit formulas for the geodesics in subRiemannian arclength parameterization. Later, an alternative expression in spatial arclength parameterization for cuspless sub-Riemannian geodesics was derived in [6, 13]. Application
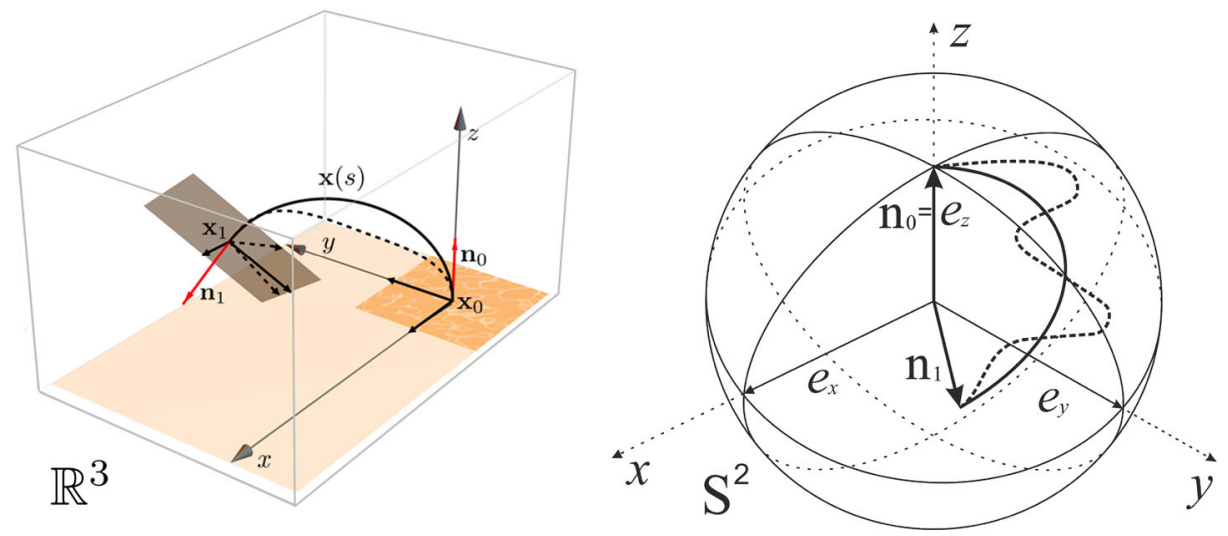

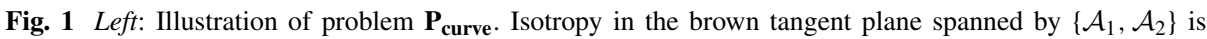
needed for a well-posed problem on the Lie group quotient $\mathrm{SE}(3) /(\{\mathbf{0}\} \times \operatorname{SO}(2))$. The tangent vectors $\mathbf{n}_{0}$ and $\mathbf{n}_{1}$ are depicted in red. Right: the angular part $\mathbf{n}(s)=\mathbf{x}^{\prime}(s)$ of the lifted curve $\left(\mathbf{x}(s), \mathbf{x}^{\prime}(s)\right) \in \mathbb{R}^{3} \times S^{2}$ 
of problem $\mathbf{P}_{\text {curve }}$ to contour completion in corrupted images was studied in [23]. The problem was also studied by Hladky and Pauls in [22] and by Ben-Yosef and Ben-Shahar in [4]. However, many imaging applications such as diffusion weighted magnetic resonance imaging (DW-MRI) require an extension to three dimensions [12, 14, 17, 31], which motivates us to study the three-dimensional curves minimizing the energy functional $\mathcal{E}(\mathbf{x})$.

\subsection{Statement of the Problem $P_{\text {curve }}$}

Let $\mathbf{x}_{0}, \mathbf{x}_{1} \in \mathbb{R}^{3}$ and $\mathbf{n}_{0}, \mathbf{n}_{1} \in S^{2}=\left\{\mathbf{v} \in \mathbb{R}^{3} \mid\|\mathbf{v}\|=1\right\}$. Our goal is to find an arc-length parameterized curve $s \mapsto \mathbf{x}(s)$ such that

$$
\begin{gathered}
\mathbf{x}=\arg \quad \inf _{\mathbf{y} \in \mathcal{C}^{\infty}\left([0, L], \mathbb{R}^{3}\right), L \geq 0,}^{\mathcal{E}(\mathbf{y}) .} \\
\mathbf{y}(0)=\mathbf{x}_{0}, \mathbf{y}^{\prime}(0)=\mathbf{n}_{0}, \\
\mathbf{y}(L)=\mathbf{x}_{1}, \mathbf{y}^{\prime}(L)=\mathbf{n}_{1} .
\end{gathered}
$$

We assume that the boundary conditions $\left(\mathbf{x}_{0}, \mathbf{n}_{0}\right)$ and $\left(\mathbf{x}_{1}, \mathbf{n}_{1}\right)$ are chosen such that a minimizer exists. Due to rotation and translational invariance of the problem, it is equivalent to the problem with the same functional and boundary conditions $\left(\mathbf{0}, \mathbf{e}_{z}\right)$ and $\left(R_{\mathbf{n}_{0}}^{T}\left(\mathbf{x}_{1}-\mathbf{x}_{0}\right), R_{\mathbf{n}_{0}}^{T} \mathbf{n}_{1}\right)$, where $\mathbf{e}_{z}$ denotes the unit vector in the $z$-axis in the right handed $\{x, y, z\}$ coordinate system and $R_{\mathbf{n}_{0}} \in \mathrm{SO}(3)$ such that $\mathbf{n}_{0}=R_{\mathbf{n}_{0}} \mathbf{e}_{z}$. Therefore, without loss of generality, we set (unless explicitly stated otherwise) $\mathbf{x}_{0}=\mathbf{0}$ and $\mathbf{n}_{0}=\mathbf{e}_{z}$ for the remainder of the article. Hence, the problem now is to find a sufficiently smooth arc-length parameterized curve $s \mapsto \mathbf{x}(s)$ such that

$$
\begin{gathered}
\mathbf{x}=\arg \quad \inf _{\mathbf{y} \in \mathcal{C}}^{\infty}\left([0, L], \mathbb{R}^{3}\right), L \geq 0, \\
\mathbf{E}(\mathbf{y}) . \\
\mathbf{y}(0)=\mathbf{0}, \mathbf{y}^{\prime}(0)=\mathbf{e}_{z}, \\
\mathbf{y}(L)=\mathbf{x}_{1}, \mathbf{y}^{\prime}(L)=\mathbf{n}_{1} .
\end{gathered}
$$

We refer to the above problem as $\mathbf{P}_{\text {curve }}$.

In this paper, we use two different parameterizations: spatial arclength $s$ and subRiemannian arclength $t(s)=\int_{0}^{s} \sqrt{\xi^{2}+\kappa^{2}(\sigma)} \mathrm{d} \sigma$. We denote the derivative $\frac{d}{d s}$ by a prime, and $\frac{d}{d t}$ by a dot.

\subsection{Structure and Results of the Article}

In Section 2, we lift problem $\mathbf{P}_{\text {curve }}$ on $\mathbb{R}^{3}$ to a sub-Riemannian problem $\mathbf{P}_{\text {mec }}$ on the quotient

$$
\mathbb{R}^{3} \rtimes S^{2}:=\mathrm{SE}(3) /(\{\mathbf{0}\} \times \mathrm{SO}(2)),
$$

where $\mathrm{SO}(2)$ is identified with all rotations in $\mathbb{R}^{3}$ about reference axis $\mathbf{e}_{z}$. Such an extension and naming ('mec' refers to mechanical) was also done for the problem $\mathbf{P}_{\text {curve }}$ on $\mathbb{R}^{2}$, cf. $[6,11]$.

To state the problem $\mathbf{P}_{\text {mec }}$ on the quotient (1.2), we first resort to the corresponding leftinvariant sub-Riemannian problem $\mathbf{P}_{\text {MEC }}$ on the Lie group SE(3). We formulate problem $\mathbf{P}_{\text {MEC }}$ in Definition 1 and problem $\mathbf{P}_{\mathbf{m e c}}$ in Definition 3.

The main result in Section 2 is Theorem 1, where we show the two requirements for sub-Riemannian geodesics $\gamma(\cdot)=(\mathbf{x}(\cdot), R(\cdot))$ in $\mathbf{P}_{\text {MEC }}$ to have the property that the corresponding spatially projected curve $\mathbf{x}(\cdot)$ is indeed a stationary curve of problem $\mathbf{P}_{\text {curve }}$. There, we also show that these sub-Riemannian geodesics in SE(3) relate to well-defined geodesics of problem $\mathbf{P}_{\text {mec }}$ on the quotient $\mathbb{R}^{3} \rtimes S^{2}$. One of the two requirements is a vanishing 
momentum component, the other is a requirement on the end-condition $\left(\mathbf{x}_{1}, \mathbf{n}_{1}=R_{\mathbf{n}_{1}} \mathbf{e}_{z}\right)$ which should belong to a set $\mathcal{R} \subset \mathbb{R}^{3} \rtimes S^{2}$ that we express as the range of an exponential map of problem $\mathbf{P}_{\text {curve }}$. In fact, this set $\mathcal{R}$ is precisely the set of end conditions for $\mathbf{P}_{\text {mec }}$ where the spatial projection of geodesics does not exhibit a cusp. The formal definition of a cusp will follow in Definition 8. For an illustration of cases $\left(\mathbf{x}_{1}, \mathbf{n}_{1}\right) \notin \mathcal{R}$ where cusps occur on the spatial projection of sub-Riemannian geodesics, see Fig. 2. The geodesic of $\mathbf{P}_{\mathbf{m e c}}$ is said to be cuspless if its spatial projection does not have a cusp. The study of cuspless sub-Riemannian geodesics in SE(3) is important for image analysis applications. In particular for the tracking of smoothly varying curvilinear structures (e.g., blood vessels or neural fibers) in noisy 3D (medical) images (see [12, 17, 31]). There, the presence of cusps in the spatial projection of a geodesic is typically undesirable, as it yields a non-smooth path in 3D images. Likewise, to the 2D case [11], it may even be used as a criterium for not connecting two given boundary conditions in an image.

In Section 3, we apply the Pontryagin maximum principle (PMP) [1, 30, 36] to problem $\mathbf{P}_{\text {MEC }}$ in Section 3.1. In Section 3.2, Theorem 2, we prove Liouville integrability. In Section 3.3, we express the canonical equations of PMP in terms of the - Cartan connection in Theorem 3. Then, a natural choice of $\mathrm{SE}(3)$ matrix representation arises in the matrix representation of the Cartan connection, i.e., the Cartan-matrix. We employ this in Theorem 4 containing one of the two key ingredients that we use for integrating the canonical equations of $\mathbf{P}_{\text {mec }}$. The other ingredient is the well-known co-adjoint orbit structure in SE(3) characterized in Lemma 1.

In Section 4, we combine the two ingredients to compute the first cusp-time (Theorem 5 in Section 4.1), and to integrate the canonical equations for geodesics of $\mathbf{P}_{\mathbf{m e c}}$. As a result, we obtain, for the first time, explicit analytic formulas for both problems $\mathbf{P}_{\text {curve }}$ and $\mathbf{P}_{\text {mec }}$. These analytic formulas involve elliptic integrals of the first and the third kind. This is summarized in Theorem 6, which is the main result of this article. Subsequently, in Section 4.3, we derive many geometric properties of the sub-Riemannian geodesics such as:

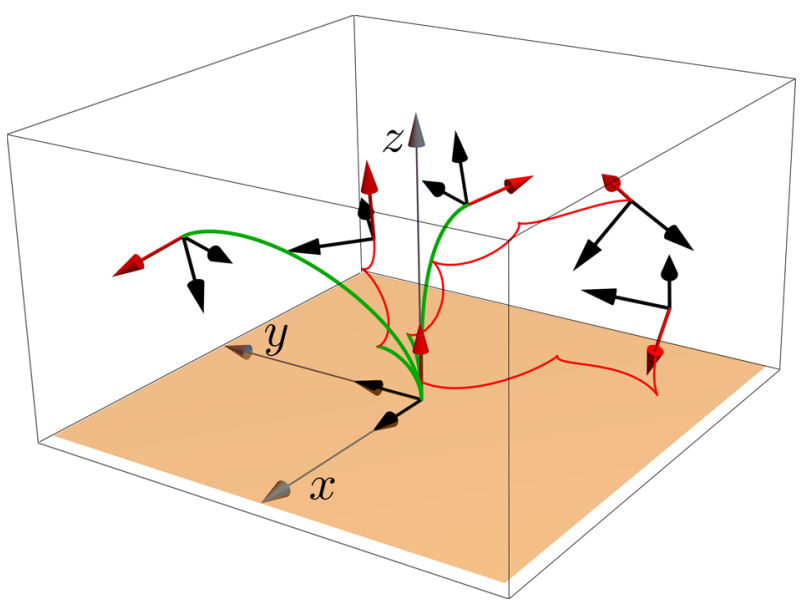

Fig. 2 The spatial projection of the geodesics of $\mathbf{P}_{\text {mec }}$ can have singularities (the cusp points). Here, the spatial projection of the geodesics of $\mathbf{P}_{\mathbf{m e c}}$ is shown in green before the first cusp point, and in red after the first cusp point. The range $\mathcal{R}$ of the exponential map of the problem $\mathbf{P}_{\text {curve }}$ consists of the end conditions reachable by the cuspless geodesic in $\mathbf{P}_{\mathbf{m e c}}$ (i.e., the end conditions reachable by only the green curves). In all cases, the end condition $\mathbf{n}_{1}=R_{\mathbf{n}_{1}} \mathbf{e}_{z}$ is depicted in red. The other black arrows show the remaining vectors $R_{\mathbf{n}_{1}} \mathbf{e}_{x}$ and $R_{\mathbf{n}_{1}} \mathbf{e}_{y}$ 
- A uniform bound on torsion of the spatial part of the geodesics in Theorem 7.

- Sufficient and necessary conditions for sub-Riemannian geodesics to be planar in Theorem 8 and Corollary 6, for which we have global optimality in Corollary 7.

- Monotony along a spatial axis (determined by the initial momentum) in Corollary 8.

- In most cases (see Corollaries 9, 10, and 11), we prove that the spatial part of subRiemannian geodesics stays in the upper half space of the initial direction (if $\mathbf{n}_{0}=\mathbf{e}_{z}$ this upper half space is $z \geq 0$ ). In particular, we prove $z \geq 0$ for all planar geodesics (Corollary 10), and $z \geq 0$ for all geodesics departing from a cusp and ending in a cusp.

- In the case of planar geodesics and/or geodesics departing from a cusp and ending in a cusp, we show in Corollaries 11 and 12 that $z=0$ can only be reached with opposite tangent $-\mathbf{e}_{z}$ via a U-shaped planar geodesic that departs from a cusp and ends in a cusp.

- The rotational and reflectional symmetries as we show in Corollary 13 in Section 4.4.

In Section 5, we conclude with numerical analysis of problem $\mathbf{P}_{\text {curve }}$ on $\mathbb{R}^{3}$. Numerical experiments in Section 5.1, see Fig. 7, indicate that the first conjugate time comes after the first cusp time, as in the 2D-case [6]. Numerical experiments in Section 5.2 on the set $\mathcal{R}$ and the cones of reachable angles, see Fig. 8, put a conjecture on homeomorphic/diffeomorphic properties on the exponential map (cf. Conjecture 1). Finally, we use the analytic formulas for the sub-Riemannian geodesics for numerical solutions to the boundary value problem as briefly explained in Section 5.3. Wolfram Mathematica code for solving the boundary value problem can be downloaded from http://bmia.bmt.tue.nl/people/RDuits/final.rar.

\section{Problem $P_{\text {curve }}$ on $\mathbb{R}^{3}, \mathbf{P}_{\text {MEC }}$ on $\mathrm{SE}(3)$, and $\mathbf{P}_{\text {mec }}$ on $\mathbb{R}^{3} \rtimes S^{2}$ and Their Connection}

In this section, we relate the problem $\mathbf{P}_{\text {curve }}$ to a sub-Riemannian problem $\mathbf{P}_{\text {mec }}$ on the quotient $\mathbb{R}^{3} \rtimes S^{2}=\mathrm{SE}(3) /(\{\mathbf{0}\} \times \mathrm{SO}(2))$, as was also done for the $\mathbf{P}_{\text {curve }}$ on $\mathbb{R}^{2}$, cf. $[6,11]$. To state the problem $\mathbf{P}_{\mathbf{m e c}}$ on this Lie group quotient, we first resort to the corresponding left-invariant sub-Riemannian problem $\mathbf{P}_{\text {MEC }}$ in the Lie group SE(3). The group $\mathrm{SE}(3)=\mathbb{R}^{3} \rtimes \mathrm{SO}(3)$ denotes the Lie group of rigid body motions on $\mathbb{R}^{3}$, which is a semidirect product $\rtimes$ of $\mathbb{R}^{3}$ and $\mathrm{SO}(3)$. An element $g \in \mathrm{SE}(3)$ is represented by the pair $(\mathbf{x}, R) \in$ $\mathbb{R}^{3} \rtimes \mathrm{SO}(3)$, and the group product is given by $g_{1} g_{2}=\left(\mathbf{x}_{1}, R_{1}\right)\left(\mathbf{x}_{2}, R_{2}\right)=\left(\mathbf{x}_{1}+R_{1} \mathbf{x}_{2}, R_{1} R_{2}\right)$, and $g^{-1}=\left(-R^{T} \mathbf{x}, R^{T}\right)$. We define sub-Riemannian problem $\mathbf{P}_{\text {MEC }}$ by means of the left-invariant frame (see Fig. 3).

The left-invariant frame consists of the following left-invariant vector fields over SE(3):

$$
\begin{aligned}
& \mathcal{A}_{1}=\cos \alpha \cos \beta \partial_{x}+(\sin \alpha \cos \gamma+\cos \alpha \sin \beta \sin \gamma) \partial_{y}+(\sin \alpha \sin \gamma-\cos \alpha \sin \beta \cos \gamma) \partial_{z}, \\
& \mathcal{A}_{2}=-\sin \alpha \cos \beta \partial_{x}+(\cos \alpha \cos \gamma-\sin \alpha \sin \beta \sin \gamma) \partial_{y}+(\cos \alpha \sin \gamma+\sin \alpha \sin \beta \cos \gamma) \partial_{z}, \\
& \mathcal{A}_{3}=\sin \beta \partial_{x}-\cos \beta \sin \gamma \partial_{y}+\cos \beta \cos \gamma \partial_{z}, \\
& \mathcal{A}_{4}=-\cos \alpha \tan \beta \partial_{\alpha}+\sin \alpha \partial_{\beta}+\cos \alpha \sec \beta \partial_{\gamma}, \\
& \mathcal{A}_{5}=\sin \alpha \tan \beta \partial_{\alpha}+\cos \alpha \partial_{\beta}-\sin \alpha \sec \beta \partial_{\gamma}, \\
& \mathcal{A}_{6}=\partial_{\alpha},
\end{aligned}
$$

where we parameterize $\mathbb{R}^{3}$ by $\{x, y, z\}$ and $\operatorname{SO}(3)$ by angles $\{\alpha, \beta, \gamma\}$ with $\alpha \in(-\pi, \pi]$, $\beta \in\left[-\frac{\pi}{2}, \frac{\pi}{2}\right]$ and $\gamma \in(-\pi, \pi]$ such that

$$
\mathrm{SO}(3) \ni R=\left(\begin{array}{ccc}
1 & 0 & 0 \\
0 & \cos \gamma & -\sin \gamma \\
0 & \sin \gamma & \cos \gamma
\end{array}\right)\left(\begin{array}{ccc}
\cos \beta & 0 & \sin \beta \\
0 & 1 & 0 \\
-\sin \beta & 0 & \cos \beta
\end{array}\right)\left(\begin{array}{ccc}
\cos \alpha & -\sin \alpha & 0 \\
\sin \alpha & \cos \alpha & 0 \\
0 & 0 & 1
\end{array}\right) \text {. }
$$





Fig. 3 The left-invariant frame representing a moving frame of reference along a curve on $\mathbb{R}^{3} \rtimes S^{2}$

Since $R \mathbf{e}_{z}=\mathbf{n}=(\sin \beta,-\sin \gamma \cos \beta, \cos \gamma \cos \beta)^{T}$, we have that $(\gamma, \beta)$ are spherical coordinates on $S^{2}$. One needs multiple charts to cover $S^{2}$. However, outside of $\pm(1,0,0)^{T}$, this choice of spherical coordinates is 1-to-1 and regular, and there it is preferable over standard Euler angles (which are singular at the unity element $\left.\mathbf{e}_{z}=(0,0,1)^{T}\right),[15, \mathrm{ch}: 2$, Fig. 4].

The corresponding co-frame is given by the co-vectors $\left\{\omega^{1}, \ldots, \omega^{6}\right\}$ satisfying

$$
\left\langle\omega^{i}, \mathcal{A}_{j}\right\rangle=\delta_{j}^{i} \text { for } i, j \in\{1, \ldots, 6\},
$$

with $\delta_{j}^{i}$ the Kronecker delta. The structure constants $c_{i, j}^{k}$ of the Lie algebra of left-invariant vector fields in $\mathrm{SE}(3)$ are given in Table 1.

We consider the sub-Riemannian manifold $\left(M, \Delta, \mathcal{G}_{\xi}\right)$, [26], with

$$
M=\operatorname{SE}(3), \Delta=\operatorname{span}\left\{\mathcal{A}_{3}, \mathcal{A}_{4}, \mathcal{A}_{5}\right\}, \text { and } \mathcal{G}_{\xi}=\xi^{2} \omega^{3} \otimes \omega^{3}+\omega^{4} \otimes \omega^{4}+\omega^{5} \otimes \omega^{5}
$$

Table 1 Table of Lie brackets $\left[\mathcal{A}_{i}, \mathcal{A}_{j}\right]=\mathcal{A}_{i} \mathcal{A}_{j}-\mathcal{A}_{j} \mathcal{A}_{i}=$ $\sum_{k=1}^{6} c_{i, j}^{k} \mathcal{A}_{k}$

\begin{tabular}{lllllll}
\hline & $\mathcal{A}_{1}$ & $\mathcal{A}_{2}$ & $\mathcal{A}_{3}$ & $\mathcal{A}_{4}$ & $\mathcal{A}_{5}$ & $\mathcal{A}_{6}$ \\
\hline $\mathcal{A}_{1}$ & 0 & 0 & 0 & 0 & $\mathcal{A}_{3}$ & $-\mathcal{A}_{2}$ \\
$\mathcal{A}_{2}$ & 0 & 0 & 0 & $-\mathcal{A}_{3}$ & 0 & $\mathcal{A}_{1}$ \\
$\mathcal{A}_{3}$ & 0 & 0 & 0 & $\mathcal{A}_{2}$ & $-\mathcal{A}_{1}$ & 0 \\
$\mathcal{A}_{4}$ & 0 & $\mathcal{A}_{3}$ & $-\mathcal{A}_{2}$ & 0 & $\mathcal{A}_{6}$ & $-\mathcal{A}_{5}$ \\
$\mathcal{A}_{5}$ & $-\mathcal{A}_{3}$ & 0 & $\mathcal{A}_{1}$ & $-\mathcal{A}_{6}$ & 0 & $\mathcal{A}_{4}$ \\
$\mathcal{A}_{6}$ & $\mathcal{A}_{2}$ & $-\mathcal{A}_{1}$ & 0 & $\mathcal{A}_{5}$ & $-\mathcal{A}_{4}$ & 0 \\
\hline
\end{tabular}


For those horizontal curves $\gamma$ (i.e., $\dot{\gamma} \in \Delta$ ) in $\operatorname{SE}(3)$ that can be parameterized by spatial arclength, one has $\int_{0}^{T} \sqrt{\left.\mathcal{G}_{\xi}\right|_{\gamma(t)}(\dot{\gamma}(t), \dot{\gamma}(t))} \mathrm{d} t=\int_{0}^{L} \sqrt{\xi^{2}+\kappa^{2}(s)} \mathrm{d} s$, which in view of Problem $\mathbf{P}_{\text {curve }}$, motivates our choice of $\left(M, \Delta, \mathcal{G}_{\xi}\right)$. Details can be found in Appendix A (A.1). The sub-Riemannian distance on $\left(\operatorname{SE}(3), \Delta, \mathcal{G}_{\xi}\right)$ is given by

$$
d(g, h)=\min _{\substack{\gamma \in \operatorname{Lip}([0, T], \operatorname{SE}(3)), T \geq 0, \dot{\gamma} \in \Delta, \gamma(0)=g, \gamma(T)=h}} \int_{0}^{T} \sqrt{\left.\mathcal{G}_{\xi}\right|_{\gamma(t)}(\dot{\gamma}(t), \dot{\gamma}(t))} \mathrm{d} t .
$$

Definition 1 In problem $\mathbf{P}_{\text {MEC }}$ on SE(3), we aim for a Lipschitzian curve $\gamma:[0, T] \rightarrow$ $\operatorname{SE}(3)$, that satisfies the boundary conditions $\gamma(0)=e:=(\mathbf{0}, I)$ and $\gamma(T)=\left(\mathbf{x}_{1}, R_{1}\right) \in$ $\mathrm{SE}(3)$, and minimizes the integral of sub-Riemannian length $\int_{0}^{T} \sqrt{\left.\mathcal{G}_{\xi}\right|_{\gamma(t)}(\dot{\gamma}(t), \dot{\gamma}(t))} \mathrm{d} t$ (with free $T$ ), and has a velocity vector $\dot{\gamma}(t) \in \Delta$ for a.e. $t \in[0, T]$.

Remark 1 As we will show in Section 3, $\mathbf{P}_{\text {MEC }}$ is well-posed and the minimizers are smooth. So one may replace $\operatorname{Lip}([0, T], \operatorname{SE}(3))$ by $\mathcal{C}^{\infty}([0, T], \operatorname{SE}(3))$ a posteriori (like we did in $\mathbf{P}_{\text {curve }}$ ).

Definition 2 A (sub-Riemannian) geodesic $\gamma$ of problem $\mathbf{P}_{\text {MEC }}$ is a horizontal curve in SE(3) (i.e. $\dot{\gamma} \in \Delta$ ) whose sufficiently short arcs are minimizers of $\mathbf{P}_{\text {MEC. }}$

Next, we will address the quotient structure (1.2) and the connection of problem $\mathbf{P}_{\text {curve }}$ on $\mathbb{R}^{3}$ to problem $\mathbf{P}_{\text {MEC }}$ on $\operatorname{SE}(3)$, and problem $\mathbf{P}_{\text {mec }}$ on $\mathbb{R}^{3} \rtimes S^{2}$.

Remark 2 Throughout this article, we identify $\mathrm{SO}(2)$ with all rotations in $\mathrm{SO}(3)$ about the reference axis, which we choose to be $\mathbf{e}_{z}$ (i.e., $\left.\mathrm{SO}(2) \equiv \mathrm{SO}(2) \oplus 1\right)$. Furthermore, $R_{\mathbf{n}}$ denotes any rotation mapping $\mathbf{e}_{z}=(0,0,1)^{T}$ onto $\mathbf{n} \in S^{2}$, whereas $R_{\mathbf{a}, \phi}$ denotes the counterclockwise rotation about axis a over angle $\phi$. The group $\operatorname{SE}(3)$ acts transitively on $\mathbb{R}^{3} \rtimes S^{2}$ by

$$
g \odot(\mathbf{y}, \mathbf{n})=(\mathbf{x}, R) \odot(\mathbf{y}, \mathbf{n})=(R \mathbf{y}+\mathbf{x}, R \mathbf{n}) .
$$

Elements in $\operatorname{SE}(3)$ that map $\left(\mathbf{0}, \mathbf{e}_{z}\right)$ to itself are denoted by

$$
h_{\alpha}:=\left(\mathbf{0}, R_{\mathbf{e}_{z}, \alpha}\right) \in\{\mathbf{0}\} \times \mathrm{SO}(2) .
$$

Regarding Eq. 1.2, we note $g_{1} \sim g_{2} \Leftrightarrow g_{1} \odot\left(\mathbf{0}, \mathbf{e}_{z}\right)=g_{2} \odot\left(\mathbf{0}, \mathbf{e}_{z}\right) \Leftrightarrow\left(g_{1}\right)^{-1} g_{2} \in$ $\{\mathbf{0}\} \times \mathrm{SO}(2)$ for all $g_{1}, g_{2} \in \mathrm{SE}(3)$. For sober notation, we write $(\mathbf{y}, \mathbf{n}) \in \mathbb{R}^{3} \rtimes S^{2}$. This represents the left coset

$$
\begin{aligned}
(\mathbf{y}, \mathbf{n}):=\left\{g \in \operatorname{SE}(3) \mid g \sim\left(\mathbf{y}, R_{\mathbf{n}}\right)\right\} & =\left\{(\mathbf{y}, R) \in \operatorname{SE}(3) \mid(\mathbf{y}, R) \odot\left(\mathbf{0}, \mathbf{e}_{z}\right)=(\mathbf{y}, \mathbf{n})\right\} \\
& =\left\{\left(\mathbf{y}, R_{\mathbf{n}} R_{\mathbf{e}_{z}, \alpha}\right) \in \operatorname{SE}(3) \mid 0 \leq \alpha<2 \pi\right\} .
\end{aligned}
$$

This is similar to the common identification $S^{2}=\mathrm{SO}(3) / \mathrm{SO}(2)$.

We obtain a well-defined distance on the quotient $\mathbb{R}^{3} \rtimes S^{2}$, recall (1.2) and (2.3), by

$$
\begin{aligned}
d_{\mathbb{R}^{3} \rtimes S^{2}}\left(\left(\mathbf{0}, \mathbf{e}_{z}\right),\left(\mathbf{y}_{1}, \mathbf{n}_{1}\right)\right) & =\min _{\alpha^{1}, \alpha^{2} \in[0,2 \pi)} d\left(e h_{\alpha^{1}},\left(\mathbf{y}_{1}, R_{\mathbf{n}_{1}}\right) h_{\alpha^{2}}\right) \\
& =\min _{\alpha^{1}, \alpha^{2} \in[0,2 \pi)} d\left(e, h_{\alpha^{1}}^{-1}\left(\mathbf{y}_{1}, R_{\mathbf{n}_{1}}\right) h_{\alpha^{2}-\alpha^{1}} h_{\alpha^{1}}\right) \\
& =\min _{\alpha \in[0,2 \pi)} d\left(e,\left(\mathbf{y}_{1}, R_{\mathbf{n}_{1}}\right) h_{\alpha}\right),
\end{aligned}
$$


where we use both left-invariance and invariance under the specific conjugations $g \mapsto$ $h_{\alpha}^{-1} g h_{\alpha}$ and where we have set $\alpha=\alpha^{2}-\alpha^{1}$. Hence, we get the following definition.

Definition 3 Problem $\mathbf{P}_{\text {mec }}$ is defined as follows on $\mathbb{R}^{3} \rtimes S^{2}$. Let $\left(\mathbf{y}_{1}, \mathbf{n}_{1}\right) \in \mathbb{R}^{3} \rtimes S^{2}$. Find

$$
[0, T] \ni t \mapsto(\mathbf{x}(t), \mathbf{n}(t))=\gamma(t) \odot\left(\mathbf{0}, \mathbf{e}_{z}\right) \in \mathbb{R}^{3} \rtimes S^{2},
$$

with $\gamma$ a Lipschitzian curve in $\operatorname{SE}(3)$ with velocity $\dot{\gamma} \in \Delta$, such that sub-Riemannian length $\int_{0}^{T} \sqrt{\left.\mathcal{G}_{\xi}\right|_{\gamma(t)}(\dot{\gamma}(t), \dot{\gamma}(t))} \mathrm{d} t$ is minimal under boundary conditions $\gamma(0)=(\mathbf{0}, I)$ and $\gamma(T)=\left(\mathbf{y}_{1}, R_{\mathbf{n}_{1}} R_{\mathbf{e}_{z}, \alpha}\right)$, where both $T \geq 0$ and $\alpha \in[0,2 \pi)$ are free variables in the optimization process.

In Section 3.1, we introduce left-invariant Hamiltonians $\lambda_{1}, \ldots, \lambda_{6}$ linear on the fibers of cotangent bundle $T^{*}(\mathrm{SE}(3))$ and apply the Pontryagin maximum principle (PMP) to the problem $\mathbf{P}_{\text {MEC }}$, where the Hamiltonian $H$ is expressed as

$$
H(\lambda)=\frac{1}{2}\left(\xi^{-2} \lambda_{3}^{2}+\lambda_{4}^{2}+\lambda_{5}^{2}\right) .
$$

To distinguish a momentum covector from its components in dual basis, we represent momentum covectors $\lambda(t)=\left.\sum_{i=1}^{6} \lambda_{i}(t) \omega^{i}\right|_{\gamma(t)}$ as row vectors $\lambda$

$$
\lambda:=\left(\lambda^{(1)}, \lambda^{(2)}\right) \text { with } \lambda^{(1)}:=\left(\lambda_{1}, \lambda_{2}, \lambda_{3}\right) \text { and } \lambda^{(2)}:=\left(\lambda_{4}, \lambda_{5}, \lambda_{6}\right),
$$

where we split $\lambda$ into a spatial part $\lambda^{(1)}$ and a rotational part $\lambda^{(2)}$.

A geodesic of problem $\mathbf{P}_{\text {mec }}$ is a curve $(\mathbf{x}(\cdot), \mathbf{n}(\cdot)) \in \mathbb{R}^{3} \rtimes S^{2}$ whose sufficiently short arcs are minimizers of $\mathbf{P}_{\text {mec }}$. First, we will show that solutions to the problem $\mathbf{P}_{\text {mec }}$ in the quotient $\mathbb{R}^{3} \rtimes S^{2}$ are geodesics in problem $\mathbf{P}_{\text {MEC }}$ in $S E(3)$ if the initial momentum $\lambda(0)$ is chosen such that $\lambda_{6}=0$.

Later on, for sub-Riemannian geodesics whose spatial projections do not exhibit cusps (see the green curves in Fig. 2), we shall rely on the spatial arclength parameter $s$ as this parametrization produces much simpler formulas. To distinguish between derivatives, we write

$$
\dot{\gamma}(t):=\frac{d}{d t} \gamma(t) \text { and } \gamma^{\prime}(s):=\frac{d}{d s} \gamma(s) .
$$

The next theorem provides us the terminal conditions of interest, the appropriate choice of representant in each equivalent class, i.e., the $\alpha$ that minimizes (2.5). In fact, this sets the choice of $\left(\mathbf{y}_{1}, R_{\mathbf{n}_{1}}\right) \in \mathrm{SE}(3)$ in $\mathbf{P}_{\text {MEC }}$ such that the spatial projection $\mathbf{x}^{*}(\cdot)$ of the optimizer of $\gamma^{*}(\cdot)=\left(\mathbf{x}^{*}(\cdot), R^{*}(\cdot)\right)$ in $\mathbf{P}_{\text {MEC }}$ coincides with the optimizer of $\mathbf{P}_{\text {curve }}$. The proof relies on notations and results in Section 3.1, but we formulate the theorem here as it fully explains the connection between problem $\mathbf{P}_{\text {curve }}$ on $\mathbb{R}^{3}$, problem $\mathbf{P}_{\text {MEC }}$ on $\operatorname{SE}(3)$, and problem $\mathbf{P}_{\text {mec }}$ on $\mathbb{R}^{3} \rtimes S^{2}$. At this point, the reader is advised to skip the proof and return to it after Section 3.1 .

Theorem 1 If the terminal point $g_{1}=\left(\mathbf{x}_{1}, R_{1}\right) \in \mathrm{SE}(3)$ is chosen such that a corresponding minimizer $\gamma^{*}$ of $\mathbf{P}_{\mathbf{M E C}}$ satisfies $^{1}$

$$
u^{3}(t):=\left\langle\left.\omega^{3}\right|_{\gamma^{*}(t)}, \dot{\gamma}^{*}(t)\right\rangle>0, \text { for all } t \in(0, T),
$$

\footnotetext{
${ }^{1}$ In Section 3, we will introduce control variables $u^{3}, u^{4}, u^{5}$ and will formulate the problem $\mathbf{P}_{\mathbf{M E C}}$ as an optimal control problem in SE(3). Moreover, it will follow from the Hamiltonian system that $\lambda_{6}$ is constant along extremals.
} 
then the minimizer $\gamma^{*}$ can be parameterized by spatial arclength $s$, and its spatial projection does not exhibit a cusp. If moreover $g_{1}$ is chosen such that $\gamma^{*}$ has vanishing momentum component

$$
\lambda_{6}(t)=\lambda_{6}(0)=0, \text { for all } t \in(0, T),
$$

then this yields the required minimum choice of $\alpha$ in Eq. 2.5, and the minimizer $\gamma^{*}(t)$ of $\mathbf{P}_{\text {MEc }}$ provides the minimizer $\left(\mathbf{x}^{*}(t), \mathbf{n}^{*}(t)\right)=\gamma^{*}(t) \odot\left(\mathbf{0}, \mathbf{e}_{z}\right)$ of problem $\mathbf{P}_{\text {mec }}$.

Under these two requirements (2.8) and (2.9), the spatial projection $\mathbf{x}^{*}(\cdot)$ of the geodesic $\gamma^{*}(\cdot)=\left(\mathbf{x}^{*}(\cdot), R^{*}(\cdot)\right)$ of problem $\mathbf{P}_{\mathbf{M E C}}$ coincides with a stationary curve of problem $\mathbf{P}_{\text {curve }}$

Proof The proof can be found in Appendix A. It relies on notation and results of Section 3. See Fig. 4 for an illustration of an explicit case.

Definition 4 Let $\widetilde{\operatorname{Exp}} p_{e}:\left\{(\lambda(0), t) \in T_{e}^{*}(\mathrm{SE}(3)) \times \mathbb{R}^{+} \mid H(\lambda(0))=\frac{1}{2}\right\} \rightarrow \operatorname{SE}(3)$ denote the exponential map of $\mathbf{P}_{\text {MEC }}$. This exponential map is the solution operator that solves the Hamiltonian system of PMP, with the Hamiltonian $H(\lambda(0))$ given by Eq. 2.6, departing from $e=(\mathbf{0}, I)$ and thereby it maps initial momentum $\lambda(0)$ and sub-Riemannian arclength $t$ onto the end-point $(\mathbf{x}(t), R(t)) \in \mathrm{SE}(3)$ of the corresponding geodesic of problem $\mathbf{P}_{\mathbf{M E C}}$.

For the required case $\lambda_{6}=0$, we will derive the operator $\widetilde{\operatorname{Exp}} p_{e}\left(\left(\lambda_{1}(0), \lambda_{2}(0), \lambda_{3}(0)\right.\right.$, $\left.\left.\lambda_{4}(0), \lambda_{5}(0), 0\right), t\right)$ explicitly in Section 4.

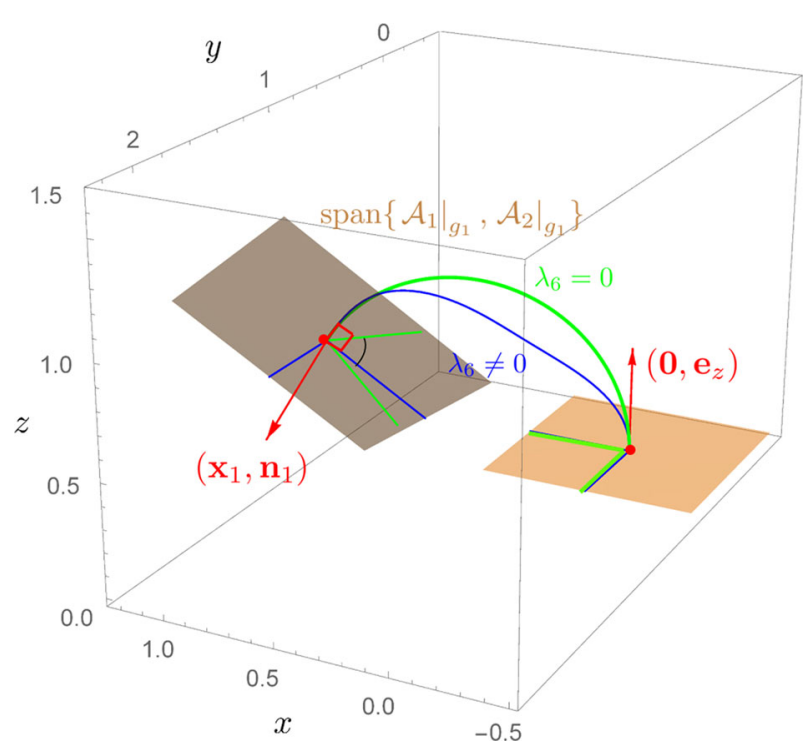

Fig. 4 End point $\left(\mathbf{x}_{1}, \mathbf{n}_{1}\right)=((0.53,1.8,1.1),(0.031,0.86,-0.51))$ in $\mathbf{P}_{\text {curve gives rise to many possible }}$

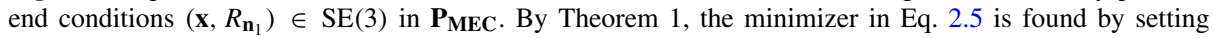
$\lambda_{6}(0)=0$. Here, the spatial projection of the minimizing geodesic is depicted in green, and the spatial projection of a geodesic with $\lambda_{6} \neq 0$ in blue. In order to show the choice of rotation $R_{\mathbf{n}_{1}} \in \mathrm{SO}(3)$, s.t. $R_{\mathbf{n}_{1}} \mathbf{e}_{z}=\mathbf{n}_{1}$, we depict the spatial frame $\left\{\left.\mathcal{A}_{1}\right|_{g_{1}},\left.\mathcal{A}_{2}\right|_{g_{1}},\left.\mathcal{A}_{3}\right|_{g_{1}}\right\}$ at the end points $g_{1}=\left(\mathbf{x}_{1}, R_{\mathbf{n}_{1}}\right)$ of both the blue and green geodesic. For the minimizing geodesic, we have $\lambda_{6}(0)=0$ and $T=d_{\mathbb{R}^{3} \rtimes S^{2}}\left(\left(\mathbf{0}, \mathbf{e}_{z}\right),\left(\mathbf{y}_{1}, \mathbf{n}_{1}\right)\right)=4$, for the other geodesic we have $\lambda_{6}(0)=2$ and indeed $T=4.65>4$ 
Definition 5 Let $\mathfrak{R}$ denote the set of boundary points $g_{1}=\left(\mathbf{x}_{1}, R_{1}\right) \in \mathrm{SE}(3)$ reachable by geodesics $\gamma^{*}$ of problem $\mathbf{P}_{\text {MEC satisfying the two requirements (2.8) and (2.9). Define }}$ $\mathcal{R}=\left\{\left(\mathbf{x}_{1}, R_{1} \mathbf{e}_{z}\right) \mid\left(\mathbf{x}_{1}, R_{1}\right) \in \mathfrak{R}\right\} \subset \mathbb{R}^{3} \rtimes S^{2}$.

Next, we formally define the exponential map of the problem $\mathbf{P}_{\text {curve }}$, where we rely on the action of $\mathrm{SE}(3)$ onto $\mathbb{R}^{3} \rtimes S^{2}$, recall (2.4).

Definition 6 The exponential map $\operatorname{Exp}: \mathcal{D}_{0} \rightarrow \mathbb{R}^{3} \rtimes S^{2}$ of $\mathbf{P}_{\text {curve }}$ is defined by:

$$
\operatorname{Exp}(\lambda(0), L):=\widetilde{\operatorname{Exp}} p_{e}(\lambda(0), T(L)) \odot\left(\mathbf{0}, \mathbf{e}_{z}\right)=\gamma^{*}(T(L)) \odot\left(\mathbf{0}, \mathbf{e}_{z}\right)=\left(\mathbf{x}^{*}(L), \frac{d}{d s} \mathbf{x}^{*}(L)\right),
$$

where $\mathbf{x}^{*}$ denotes the minimizer of $\mathbf{P}_{\text {curve }}, \gamma^{*}$ denotes a minimizer of $\mathbf{P}_{\mathbf{M E C}}$, and with domain

$$
\begin{aligned}
& \mathcal{D}_{0}=\left\{(\lambda(0), L) \in \mathcal{D} \mid \lambda_{6}(0)=0\right\}, \text { with } \\
& \mathcal{D}=\left\{(\lambda(0), L) \in T_{e}^{*}(\operatorname{SE}(3)) \times \mathbb{R}^{+} \mid H(\lambda(0))=\frac{1}{2}, L \leq s_{\text {max }}(\lambda(0)), \lambda^{(1)}(0) \neq \mathbf{0}\right\},
\end{aligned}
$$

where $s_{\max }(\lambda(0)) \in \mathbb{R} \cup\{\infty\}$ is the maximal length of the spatial projection $\mathbf{x}^{*}$ of the sub-Riemannian geodesic $\gamma^{*}$ to the first cusp.

We exclude $\lambda^{(1)}(0)=\mathbf{0}$ from the domain $\mathcal{D}$ to avoid pure rotations which are not solutions to the problem $\mathbf{P}_{\text {curve }}$. This is also done in the SE(2) case [11, Remark 5.5]. By Theorem 1, we have the following result.

Corollary 1 The set $\mathcal{R}$ equals the range of the exponential map of $\mathbf{P}_{\text {curve: }}$

$$
\mathcal{R}=\left\{\operatorname{Exp}(\lambda(0), L) \mid(\lambda(0), L) \in \mathcal{D}_{0}\right\} \subset \mathbb{R}^{3} \rtimes S^{2},
$$

i.e., it coincides with all possible end conditions $\left(\mathbf{x}(L), \mathbf{x}^{\prime}(L)\right)$ of geodesics of $\mathbf{P}_{\mathbf{c u r v e}}$.

Definition 7 An end condition $\left(\mathbf{x}_{1}, \mathbf{n}_{1}\right) \in \mathbb{R}^{3} \rtimes S^{2}$ is called admissible if $\left(\mathbf{x}_{1}, \mathbf{n}_{1}\right) \in \mathcal{R}$.

\section{PMEC: Sub-Riemannian Problem on SE(3)}

In this section, we study the problem $\mathbf{P}_{\text {MEC }}$. The optimal control theoretical formulation of this sub-Riemannian problem is to find a Lipschitzian curve $\gamma:[0, T] \rightarrow \operatorname{SE}(3)$, with boundary conditions $\gamma(0)=e:=(\mathbf{0}, I)$ and $\gamma(T)=\left(\mathbf{x}_{1}, R_{1}\right) \in \operatorname{SE}(3)$, such that

$$
\begin{aligned}
& \int_{0}^{T} \sqrt{\left.\mathcal{G}_{\xi}\right|_{\gamma(t)}(\dot{\gamma}(t), \dot{\gamma}(t))} \mathrm{d} t=\int_{0}^{T} \sqrt{\xi^{2}\left(u^{3}(t)\right)^{2}+\left(u^{4}(t)\right)^{2}+\left(u^{5}(t)\right)^{2}} \mathrm{~d} t \rightarrow \min \text { (with free } T \text { ), } \\
& \text { with } \dot{\gamma}(t)=\left.\sum_{i=3}^{5} u^{i}(t) \mathcal{A}_{i}\right|_{\gamma(t)}=\left.\sum_{i=3}^{5}\left\langle\left.\omega^{i}\right|_{\gamma(t)}, \dot{\gamma}(t)\right\rangle \mathcal{A}_{i}\right|_{\gamma(t)},
\end{aligned}
$$

where the control variables $u^{i} \in \mathbb{L}_{\infty}([0, T])$ for $i=3$, 4, 5. In particular, we only consider the curves for which the absolute curvature of the spatial projections is in $\mathbb{L}_{\infty}([0, T])$. The control variables are contravariant components of the velocity vector, so we index them with upper indices.

Remark 3 The problem $\mathbf{P}_{\text {MEC }}$ given by Eq. 3.1 can be seen as a motion planing problem for a spacecraft, that can move forward/backward (in direction $\mathcal{A}_{3}$ ) and rotate about axis 
$u^{4} \mathcal{A}_{1}+u^{5} \mathcal{A}_{2}$. The control $u^{3}$ determines spacial velocity (the sign of $u^{3}$ determines forward/backward propagation), while the controls $u^{4}$ and $u^{5}$ determine the angular velocity, cf. Fig. 3.

The existence of minimizers for the problem $\mathbf{P}_{\text {MEC }}$ is guaranteed by the theorems by Chow-Rashevskii and Filippov on sub-Riemannian structures [1].

Remark 4 About smoothness of minimizers of $\mathbf{P}_{\text {MEC }}$.

- We have that for $\mathbf{P}_{\text {MEC }}$, there are no abnormal extremals. It follows from the fact that any sub-Riemannian manifold with a two-generating distribution does not allow abnormal extremals (see Chapter 20.5.1 in [1]). This is the case here as we have for $\Delta:=\left\{\mathcal{A}_{3}, \mathcal{A}_{4}, \mathcal{A}_{5}\right\}, \operatorname{dim}([\Delta, \Delta])=\operatorname{dim}\left(\operatorname{span}\left\{\mathcal{A}_{1}, \mathcal{A}_{2}, \mathcal{A}_{3}, \mathcal{A}_{4}, \mathcal{A}_{5}, \mathcal{A}_{6}\right\}\right)=$ $\operatorname{dim}(\mathrm{SE}(3))=6$.

- Due to the non-existence of abnormal extremals, the geodesics are always analytic [1] and so are the extremal controls. A priori, in the application of the Pontryagin maximum principle (PMP) [1, 36], one cannot restrict to smooth controls where one relies on $\mathbb{L}_{\infty}$-controls for $\mathbf{P}_{\text {MEC }}$ (and $\mathbb{L}_{1}$-controls for $\mathbf{P}_{\text {curve }}$, similar to the $\operatorname{SE}(2)$-case [6, ch:5.1, App.B]). In retrospect, however, the minimizers are analytic, and one can write the sub-Riemannian distance from $g$ to $e=(\mathbf{0}, I)$ on $\left(\operatorname{SE}(3), \Delta, \mathcal{G}_{\xi}\right)$ as

$$
d(g, e)=\min _{\substack{\gamma \in C^{\infty}([0, T], \operatorname{SE}(3)), T \geq 0, \dot{\gamma} \in \Delta, \gamma(0)=e, \gamma(T)=g}} \int_{0}^{T} \sqrt{\left.\mathcal{G}_{\xi}\right|_{\gamma(t)}(\dot{\gamma}(t), \dot{\gamma}(t))} \mathrm{d} t .
$$

Next, in the application of PMP to the problem $\mathbf{P}_{\mathbf{M E C}}$, we rely on the sub-Riemannian arclength parameter $t$, which is well-defined for all SR-geodesics in $\left(\operatorname{SE}(3), \Delta, \mathcal{G}_{\xi}\right)$. Before a cusp occurs, recall Fig. 2, one can also use spatial arclength parameterization $s$, related by $t(s)=\int_{0}^{s} \sqrt{\xi^{2}+\kappa^{2}(\sigma)} \mathrm{d} \sigma$. The formal definition of a cusp time is given bellow.

Definition 8 A cusp time is a time $0<t_{\text {cusp }}<T$ when the third control component $u^{3}(t)$ (responsible for spatial propagation in $\mathbf{P}_{\text {MEC }}$ ) vanishes, $u^{3}\left(t_{c u s p}\right)=0$ and moreover $\dot{u}^{3}\left(t_{\text {cusp }}\right) \neq 0$.

For illustrations of cusps, see Fig. 2 and for planar sub-Riemannian geodesics see [11, Fig. 2], [5].

\subsection{Application of Pontryagin Maximum Principle}

A first-order necessary optimality condition is given by PMP [1, 30]. Note that PMP gives a necessary but not a sufficient condition of optimality. Geodesics of $\mathbf{P}_{\text {MEC }}$ loses local optimality after the first conjugate point and global optimality after the first Maxwell point (see [1]).

The Cauchy-Schwarz inequality implies that the minimization problem for the subRiemannian length functional is equivalent to the minimization problem for the action functional (see [1])

$$
J=\frac{1}{2} \int_{0}^{T}\left(\xi^{2}\left(u^{3}\right)^{2}+\left(u^{4}\right)^{2}+\left(u^{5}\right)^{2}\right) \mathrm{d} t \rightarrow \min , \quad \text { with fixed } T>0 .
$$


Next, we apply PMP to problem $\mathbf{P}_{\text {MEC }}$ using the equivalent action functional, i.e., to the following optimal control problem:

$$
\dot{\gamma}(t)=\left.\sum_{i=3}^{5} u^{i}(t) \mathcal{A}_{i}\right|_{\gamma(t)}, \quad J \rightarrow \min , \quad \gamma(0)=e, \gamma(T)=g_{1} \in \operatorname{SE}(3) .
$$

Denote $q=(x, y, z, \gamma, \beta, \alpha) \in \mathbb{R}^{3} \times(-\pi, \pi] \times\left[-\frac{\pi}{2}, \frac{\pi}{2}\right] \times(-\pi, \pi]$ which is identified with an element from SE(3) via (2.1). The natural momentum coordinates $\left\{\lambda_{i}\right\}$ for left-invariant sub-Riemannian problems come along with the left-invariant Hamiltonians $h_{i}: T^{*}(\mathrm{SE}(3)) \rightarrow \mathbb{R}$ (see [1]), given by

$$
h_{i}(q, \lambda)=\left\langle\lambda(q),\left.\mathcal{A}_{i}\right|_{q}\right\rangle=\lambda_{i}(q), \quad i=1, \ldots 6,
$$

where $\lambda(q)=\left.p_{1}(q) \mathrm{d} x\right|_{q}+\left.p_{2}(q) \mathrm{d} y\right|_{q}+\left.p_{3}(q) \mathrm{d} z\right|_{q}+\left.p_{4}(q) \mathrm{d} \gamma\right|_{q}+\left.p_{5}(q) \mathrm{d} \beta\right|_{q}+\left.p_{6}(q) \mathrm{d} \alpha\right|_{q}=$ $\left.\sum_{i=1}^{6} \lambda_{i}(q) \omega^{i}\right|_{q} \in T_{q}^{*}(\mathrm{SE}(3))$ denotes a momentum covector expressed in respectively the fixed and the moving dual frame.

Now, we apply PMP. By Remark 4, we only need to consider the normal case. The control-dependent Hamiltonian reads $H_{u}=u^{3} \lambda_{3}+u^{4} \lambda_{4}+u^{5} \lambda_{5}-$ $\frac{1}{2}\left(\xi^{2}\left(u^{3}\right)^{2}+\left(u^{4}\right)^{2}+\left(u^{5}\right)^{2}\right)$. Optimization over all controls produces the (maximized) Hamiltonian

$$
H=\max _{u \in \mathbb{R}^{3}} H_{u}=\frac{1}{2}\left(\xi^{-2} \lambda_{3}^{2}+\lambda_{4}^{2}+\lambda_{5}^{2}\right),
$$

and gives expression for the extremal controls

$$
u^{3}=\frac{\lambda_{3}}{\xi^{2}}, \quad u^{4}=\lambda_{4}, \quad u^{5}=\lambda_{5} .
$$

By virtue of the Lie brackets (see Table 1), we have the Poisson brackets

$$
\begin{aligned}
& \left\{H, \lambda_{1}\right\}=-\lambda_{3} \lambda_{5}, \quad\left\{H, \lambda_{2}\right\}=\lambda_{3} \lambda_{4}, \quad\left\{H, \lambda_{3}\right\}=\lambda_{1} \lambda_{5}-\lambda_{2} \lambda_{4}, \\
& \left\{H, \lambda_{4}\right\}=\frac{\lambda_{2} \lambda_{3}}{\xi^{2}}-\lambda_{5} \lambda_{6}, \quad\left\{H, \lambda_{5}\right\}=\lambda_{4} \lambda_{6}-\frac{\lambda_{1} \lambda_{3}}{\xi^{2}}, \quad\left\{H, \lambda_{6}\right\}=0 .
\end{aligned}
$$

Thus, the Hamiltonian system of PMP reads as follows:

$$
\left\{\begin{array}{l}
\dot{\lambda}_{1}=-\lambda_{3} \lambda_{5}, \\
\dot{\lambda}_{2}=\lambda_{3} \lambda_{4}, \\
\dot{\lambda}_{3}=\lambda_{1} \lambda_{5}-\lambda_{2} \lambda_{4}, \\
\dot{\lambda}_{4}=\frac{\lambda_{2} \lambda_{3}}{\xi^{2}}-\lambda_{5} \lambda_{6}, \\
\dot{\lambda}_{5}=\lambda_{4} \lambda_{6}-\frac{\lambda_{1} \lambda_{3}}{\xi^{2}}, \\
\dot{\lambda}_{6}=0,
\end{array}\right.
$$$$
\left\{\begin{array}{l}
\dot{x}=\frac{\lambda_{3}}{\xi^{2}} \sin \beta, \\
\dot{y}=-\frac{\lambda_{3}}{\xi^{2}} \cos \beta \sin \gamma, \\
\dot{z}=\frac{\lambda_{3}}{\xi^{2}} \cos \beta \cos \gamma, \\
\dot{\gamma}=\sec \beta\left(\lambda_{4} \cos \alpha-\lambda_{5} \sin \alpha\right), \\
\dot{\beta}=\lambda_{4} \sin \alpha+\lambda_{5} \cos \alpha, \\
\dot{\alpha}=-\left(\lambda_{4} \cos \alpha-\lambda_{5} \sin \alpha\right) \tan \beta,
\end{array}\right.
$$

— vertical part (for adjoint variables), — horizontal part (for state variables).

These equations only hold outside the singularities of the angles chart $\{\alpha, \beta, \gamma\}$. In particular, if $\beta \in\left\{-\frac{\pi}{2}, \frac{\pi}{2}\right\}$, we can rely on standard Euler angles $R=R_{\mathbf{e}_{z}, \bar{\gamma}} R_{\mathbf{e}_{y}, \bar{\beta}} R_{\mathbf{e}_{z}, \bar{\alpha}}$ and in the vicinity of those singularities, the final three equations of the horizontal part need to be replaced by

$$
\dot{\bar{\gamma}}=-\frac{\cos \bar{\alpha}}{\sin \bar{\beta}} \lambda_{4}+\frac{\sin \bar{\alpha}}{\sin \bar{\beta}} \lambda_{5}, \dot{\bar{\beta}}=\lambda_{4} \sin \bar{\alpha}+\lambda_{5} \cos \bar{\alpha}, \dot{\bar{\alpha}}=\left(\lambda_{4} \cos \bar{\alpha}-\lambda_{5} \sin \bar{\alpha}\right) \cot \bar{\beta} .
$$

The sub-Riemannian geodesics are solutions to the Hamiltonian system. Finding a parameterization of the sub-Riemannian geodesics is a nontrivial problem. In order to 
guarantee that such a parametrization exists, we first prove Liouville integrability of the system. Here, we follow the same approach as in [24].

The next remark shows that we can restrict ourselves to the case $\xi=1$.

Remark 5 The Hamiltonian system of PMP (3.5) reveals the scaling homothety. The case $\xi \neq 1$ is obtained from the case $\xi=1$ by

$$
\lambda \mapsto \lambda\left(\Lambda_{\xi} \oplus I_{3}\right) \text { and }(\mathbf{x}, R) \mapsto\left(\Lambda_{\xi}^{-1} \mathbf{x}, R\right),
$$

with $\Lambda_{\xi}=\operatorname{diag}\{\xi, \xi$, $\xi\}$. So in order to obtain solution $\mathbf{x}^{*}$ of $\mathbf{P}_{\text {curve }}$ with boundary conditions $\left(\mathbf{x}(0), \mathbf{x}^{\prime}(0)\right)=\left(\mathbf{0}, \mathbf{e}_{z}\right)$ and $\left(\mathbf{x}(L), \mathbf{x}^{\prime}(L)\right)=\left(\mathbf{x}_{1}, \mathbf{n}_{1}\right)$ for $\xi>0$, we first solve $\mathbf{P}_{\text {curve }}$ with boundary conditions $\left(\mathbf{x}(0), \mathbf{x}^{\prime}(0)\right)=\left(\mathbf{0}, \mathbf{e}_{z}\right)$ and $\left(\mathbf{x}(L), \mathbf{x}^{\prime}(L)\right)=\left(\xi \mathbf{x}_{1}, \mathbf{n}_{1}\right)$ for $\xi=1$ in dynamics (3.5). Then the optimal curve $\mathbf{x}(\cdot)$ needs to be scaled back $\mathbf{x}^{*}(s)=\xi^{-1} \mathbf{x}(s)$. The homothety boils down to making the problem dimensionless, as the physical dimension of $\xi^{-1}$ is spatial length.

Remark 6 All prerequisites for the proof of Theorem 1 are now given. See Appendix A.

\subsection{Liouville Integrability}

To prove the Liouville integrability of the Hamiltonian system (3.5), one should construct a complete system of first integrals, i.e., indicate six first integrals in involution (w.r.t. Poisson brackets) and functionally independent on an open dense domain in $T^{*}(\operatorname{SE}(3))$ [2, p. 107].

It is well known that the Hamiltonian $H=\frac{1}{2}\left(\lambda_{3}^{2}+\lambda_{4}^{2}+\lambda_{5}^{2}\right)$ is a first integral of the Hamiltonian system. From the vertical part of Eq. 3.5, we can immediately see one more first integral $\lambda_{6}$, which is functionally independent from $H$. Since $\left\{H, \lambda_{6}\right\}=0$, we see that the integrals $H$ and $\lambda_{6}$ are in involution. The Hamiltonian system directly reveals the first integral $W=-\lambda_{1} \lambda_{4}-\lambda_{2} \lambda_{5}-\lambda_{3} \lambda_{6}$. This integral is a Casimir function, i.e., $\left\{W, \lambda_{i}\right\}=0, i=1, \ldots, 6$. Casimir functions are functions on the dual space of the Lie algebra commuting in the sense of Poisson brackets with all left-invariant Hamiltonians. They are universal conservation laws on the Lie group. Connected joint level surfaces of all Casimir functions are coadjoint orbits (see [20, Prop. 7.7]). Since these orbits are always even-dimensional (they are symplectic manifolds), the difference between the dimension of the Lie group and the number of Casimir functions is even. Casimir functions are found by solving $\left\{C, \lambda_{i}\right\}=0$. For polynomial functions $C$, we can write $C$ with undetermined coefficients as a polynomial of degree $1,2,3$, and solve the resulting system of equations algebraically. The second Casimir function in $\operatorname{SE}(3)$ is given by $\mathfrak{c}^{2}=\lambda_{1}^{2}+\lambda_{2}^{2}+\lambda_{3}^{2}$. For details on the Casimir functions, see the work of A.A. Kirillov [19] or the book of V. Jurdjevic [18].

To construct the complete system of first integrals, we consider integrals $H, \lambda_{6}$ and $W$ and find three more first integrals. Then we show that all six integrals are functionally independent on an open dense domain in $T^{*}(\mathrm{SE}(3))$ and are in involution.

\subsubsection{Right-Invariant Hamiltonians}

For any right-invariant vector field $\mathcal{B} \in \operatorname{VEC}(\operatorname{SE}(3))$, one can define the corresponding Hamiltonian $\rho(q, \lambda)=\left\langle\lambda(q),\left.\mathcal{B}\right|_{q}\right\rangle, \lambda(q) \in T_{q}^{*}(\operatorname{SE}(3))$. Since right translations commute with the left ones, left-invariant vector fields commute with right-invariant ones. Thus, leftinvariant Hamiltonians Poisson-commute with the right-invariant ones. 
The right-invariant vector fields are given by

$$
\begin{array}{r}
\mathcal{B}_{1}=-\partial_{x}, \quad \mathcal{B}_{2}=-\partial_{y}, \quad \mathcal{B}_{3}=-\partial_{z}, \quad \mathcal{B}_{4}=z \partial_{y}-y \partial_{z}-\partial_{\gamma}, \\
\mathcal{B}_{5}=-z \partial_{x}+x \partial_{z}-\sin \gamma \sec \beta \partial_{\alpha}-\cos \gamma \partial_{\beta}-\sin \gamma \tan \beta \partial_{\gamma}, \\
\mathcal{B}_{6}=y \partial_{x}-x \partial_{y}-\cos \gamma \sec \beta \partial_{\alpha}-\sin \gamma \partial_{\beta}+\cos \gamma \tan \beta \partial_{\gamma} .
\end{array}
$$

Then, the right-invariant Hamiltonians are defined by $\rho_{i}(q, \lambda)=\left\langle\lambda(q),\left.\mathcal{B}_{i}\right|_{q}\right\rangle$. Since the table of Poisson brackets between $\rho_{i}$ coincides with the commutator of corresponding vector fields (cf. Table 1 ), we see that only $\rho_{1}, \rho_{2}$, and $\rho_{3}$ are in involution. Their expression via the left-invariant Hamiltonian $\lambda_{i}$ reads as

$$
\begin{aligned}
& \rho_{1}=-\lambda_{1} \cos \alpha \cos \beta+\lambda_{2} \cos \beta \sin \alpha-\lambda_{3} \sin \beta, \\
& \rho_{2}=-\cos \gamma\left(\lambda_{2} \cos \alpha+\lambda_{1} \sin \alpha\right)+\left(\lambda_{3} \cos \beta+\left(-\lambda_{1} \cos \alpha+\lambda_{2} \sin \alpha\right) \sin \beta\right) \sin \gamma, \\
& \rho_{3}=-\lambda_{3} \cos \beta \cos \gamma+\cos \gamma\left(\lambda_{1} \cos \alpha-\lambda_{2} \sin \alpha\right) \sin \beta-\left(\lambda_{2} \cos \alpha+\lambda_{1} \sin \alpha\right) \sin \gamma .
\end{aligned}
$$

\subsubsection{Independence of Integrals}

In order to study the functional independence of the integrals $H, \lambda_{6}, W, \rho_{1}, \rho_{2}, \rho_{3}$ at a point $(q, \lambda) \in T^{*}(\operatorname{SE}(3))$, introduce the Jacobian matrix

$$
J(q, \lambda)=\left.\left(\nabla H\left|\nabla \lambda_{6}\right| \nabla W\left|\nabla \rho_{1}\right| \nabla \rho_{2} \mid \nabla \rho_{3}\right)^{T}\right|_{(q, \lambda)} .
$$

Liouville integrability of the Hamiltonian system follows by the study of the vertical derivatives of the integrals (i.e., the derivatives w.r.t. the variables $\lambda_{i}$ ). By analyticity, functional independence of the integrals on an open dense domain in $T^{*}(\operatorname{SE}(3))$ follows from linear independence of gradients of the integrals at a single point $(q, \lambda) \in T^{*}(\operatorname{SE}(3))$. Since we have

$$
\frac{\partial\left(\rho_{1}, \rho_{2}, \rho_{3}, W, H, \lambda_{6}\right)}{\partial\left(\lambda_{1}, \lambda_{2}, \lambda_{3}, \lambda_{4}, \lambda_{5}, \lambda_{6}\right)}(q, \lambda)=-\lambda_{2} \lambda_{4}+\lambda_{1} \lambda_{5} \not \equiv 0,
$$

the first integrals $\rho_{1}, \rho_{2}, \rho_{3}, I, H, \lambda_{6}$ are functionally independent. Here, we use that $-\lambda_{2} \lambda_{4}+\lambda_{1} \lambda_{5}=\dot{\lambda}_{3}=\dot{u}^{3}=\frac{d^{2} s}{d t^{2}} \not \equiv 0$. So we proved the following theorem.

Theorem 2 The Hamiltonian system (3.5) is Liouville integrable. Functionally independent first integrals are $\rho_{1}, \rho_{2}, \rho_{3}, c f$. (3.6), $W=-\lambda_{1} \lambda_{4}-\lambda_{2} \lambda_{5}-\lambda_{3} \lambda_{6}, H=\frac{1}{2}\left(\lambda_{3}^{2}+\lambda_{4}^{2}+\lambda_{5}^{2}\right)$ and $\lambda_{6}$.

Explicit integration of Eq. 3.5 in sub-Riemannian arclength parametrization $t$ is a difficult problem. Further in Section 4, we show that using spatial arclength parametrization $s$ leads to relatively simple expressions for sub-Riemannian geodesics whose spatial projections do not exhibit cusps.

\subsection{The - Cartan Connection $\bar{\nabla}$}

In the sub-Riemannian manifold $\left(\operatorname{SE}(3), \Delta, \mathcal{G}_{\xi}\right)$, the directions $\mathcal{A}_{1}, \mathcal{A}_{2}$, and $\mathcal{A}_{6}$ are prohibited in the tangent bundle. To get a better grasp on what this means on the manifold level, we consider principal fibre bundles. We use the minus '-' Cartan connection [8] to connect the Hamiltonian PMP approach to the Lagrangian reduction approach by Bryant and Griffiths [7]. It provides more intuition and is an important tool toward explicit formulas. As is seen by the following theorem, these curves actually describe parallel transport of the 
momentum covectors w.r.t. Cartan connections. In the original Cartan and Schouten article [8], three Cartan connections are presented, the + , the - , and 0 connection. Here, we shall rely on the minus - Cartan connection for which the left-invariant vector fields are autoparallel [27], since our geometrical control problem is expressed in left-invariant vector fields. In order to keep track of correct tensorial computations, we deal with the general case $\xi>0$ in Theorem 3. However, by Remark 5, only the case $\xi=1$ is considered in the remainder of the article.

Definition 9 We define connection $\bar{\nabla}$ on the (horizontal) tangent bundle of ( $\operatorname{SE}(3), \Delta, \mathcal{G}_{\xi}$ ) by

$$
\bar{\nabla}_{\dot{\gamma}} \mathcal{A}:=\sum_{k=3}^{5}\left(\left(\dot{a}^{k}\right)-\sum_{i, j=3}^{5} c_{i, j}^{k}\left(\dot{\gamma}^{i}\right) a^{j}\right) \mathcal{A}_{k},
$$

with $\dot{\gamma}=\left.\sum_{i=3}^{5} \dot{\gamma}^{i} \mathcal{A}_{i}\right|_{\gamma}, \mathcal{A}=\sum_{k=3}^{5} a^{k} \mathcal{A}_{k}$ and Lie algebra structure constants $c_{i, j}^{k}$ given in Table 1.

It is a partial - Cartan connection that originates from a specific choice of principle fiber bundle. For details, see Appendix B below. Another ingredient in the theorem below is the standard exponential map from Lie algebra to Lie group given by $T_{(\mathbf{0}, I)}(\mathrm{SE}(3)) \ni A \rightarrow$ $e^{A} \in \mathrm{SE}(3)$.

Theorem 3 Horizontal exponential curves in $\left(\mathrm{SE}(3), \Delta, \mathcal{G}_{\xi}\right)$, are given by $t \mapsto$ $g_{0} e^{t \sum_{i=3}^{5} c^{i} A_{i}}$, with $\xi^{2}\left(c^{3}\right)^{2}+\left(c^{4}\right)^{2}+\left(c^{5}\right)^{2}=1, g_{0} \in \mathrm{SE}(3)$, and they are precisely the auto-parallel curves, i.e.,

$$
\bar{\nabla}_{\dot{\gamma}} \dot{\gamma}=0 \text {. }
$$

Along sub-Riemannian geodesics in $\left(\mathrm{SE}(3), \Delta, \mathcal{G}_{\xi}\right)$ one has covariantly constant momentum, i.e.,

$$
\bar{\nabla}_{\dot{\gamma}}^{*} \lambda:=\sum_{i=1}^{6}\left(\dot{\lambda}_{i}+\sum_{j=3}^{5} \sum_{k=1}^{6} c_{i, j}^{k} \lambda_{k} \dot{\gamma}^{j}\right) \omega^{i}=0 .
$$

When setting contravariant components $\lambda^{i}=\xi^{i} \lambda_{i}, \xi^{3}=\xi^{-2}, \xi^{4}=\xi^{5}=1$, and $P_{\Delta^{*}} \lambda=\sum_{i=3}^{5} \lambda_{i} \omega^{i}$ then the Hamiltonian system of PMP (3.5) can be written as:

$$
\begin{aligned}
& \forall_{i \in\{1, \ldots, 6\}}: \dot{\lambda}_{i}+\sum_{j=3}^{5} \sum_{k=1}^{6} c_{i, j}^{k} \lambda_{k} \lambda^{j}=0 \text { and } \forall_{i \in\{3,4,5\}}: \dot{\gamma}^{i}=\lambda^{i} \\
& \text { (vertical part) (horizontal part), }
\end{aligned}
$$

which is equivalent to $\bar{\nabla}_{\dot{\gamma}}^{*} \lambda=0$ and $\mathcal{G}_{\xi}^{-1} P_{\Delta^{*} \lambda}=\dot{\gamma}$.

Proof Define $\dot{\gamma}^{i}:=\left\langle\left.\omega^{i}\right|_{\gamma}, \dot{\gamma}\right\rangle$. Note that $\dot{\gamma}^{i}=u^{i}$. Here, we write $\dot{\gamma}^{i}$ (instead of $u^{i}$ ) to stress the curve dependence of the control components. Then following the same approach as done in [13], [11, App.C] (for the case SE(2)), the Cartan connection on the tangent bundle is given by Eq. 3.7. From Eq. 3.7, we see that the auto-parallel curves are horizontal exponential curves: 
$\bar{\nabla}_{\dot{\gamma}} \dot{\gamma}=0 \Leftrightarrow \forall_{i \in\{3,4,5\}} \ddot{\gamma}^{i}=0 \Leftrightarrow \forall_{i \in\{3,4,5\}} \dot{\gamma}^{i}=u^{i}=c^{i}$ for some constants $c^{i} \Leftrightarrow \gamma(t)=$ $t \sum_{i=3}^{5} c^{i} A_{i}$

$\gamma(0) e^{i=3}$, and to ensure $t$ to be the sub-Riemannian arclength parameter we must have $\xi^{2}\left(c^{3}\right)^{2}+\left(c^{4}\right)^{2}+\left(c^{5}\right)^{2}=1$. Now the partial ('right' or - ) Cartan connection $\bar{\nabla}$ on the tangent bundle naturally imposes the partial Cartan connection $\bar{\nabla}^{*}$ on the cotangent bundle, as follows:

$$
\left.\bar{\nabla}_{\dot{\gamma}}^{*} \sum_{i=1}^{6} \lambda_{i} \omega^{i}\right|_{\gamma}=\left.\sum_{i=1}^{6}\left(\dot{\lambda}_{i}+\sum_{j=3}^{5} \sum_{k=1}^{6} c_{i, j}^{k} \lambda_{k} \dot{\gamma}^{j}\right) \omega^{i}\right|_{\gamma},
$$

which follows from Eq. 3.7 and $\mathrm{d}\left\langle\left.\omega^{k}\right|_{\gamma},\left.\mathcal{A}_{j}\right|_{\gamma}\right\rangle=\left\langle\left.\bar{\nabla}_{\dot{\gamma}}^{*} \omega^{k}\right|_{\gamma},\left.\mathcal{A}_{j}\right|_{\gamma}\right\rangle+\left\langle\left.\omega^{k}\right|_{\gamma},\left.\bar{\nabla}_{\dot{\gamma}} \mathcal{A}_{j}\right|_{\gamma}\right\rangle=0$, and $c_{i, j}^{k}=-c_{j, i}^{k}$. Now, by the horizontal part of PMP, we have $\dot{\gamma}^{i}=\lambda^{i}$ for all $i \in\{3,4,5\}$, so that the result follows by substituting this equality into Eq. 3.9.

Next, we switch to spatial arclength parameter $s$, as this is convenient. Recall $\frac{d}{d s}$ is denoted by a prime. Also recall that $s$-parametrization is well defined until the spatial projection of a sub-Riemannian geodesic exhibits a cusp (recall Definition 8).

Denote by $s_{\max }$ the minimum positive value of the parameter $s$ where such a cusp appears. Explicit expression for $s_{\max }$ in terms of the initial momentum $\lambda(0)$ will follow (see Eq. 4.5). Next, to find the sub-Riemannian geodesics, we integrate the equation in Theorem 3 via a suitable matrix representation visible in the Cartan-matrix of the Cartan connection. Such a group representation $m: \operatorname{SE}(3) \rightarrow \operatorname{Aut}\left(\mathbb{R}^{6}\right)$ is given by

$$
m(\mathbf{x}, R):=\left(\begin{array}{cc}
R & \sigma_{\mathbf{x}} R \\
0 & R
\end{array}\right),
$$

where $\sigma_{\mathbf{x}}=\sum_{i=1}^{3} x^{i} A_{3+i} \in \operatorname{so}(3)$, so that $\sigma_{\mathbf{x}} \mathbf{y}=\mathbf{x} \times \mathbf{y}$, with $\mathbf{x}=\sum_{i=1}^{3} x^{i} \mathbf{e}_{i}$ and $A_{3+i} \in \operatorname{so(3)}$ given by

$$
A_{4}=\left(\begin{array}{ccc}
0 & 0 & 0 \\
0 & 0 & -1 \\
0 & 1 & 0
\end{array}\right), \quad A_{5}=\left(\begin{array}{ccc}
0 & 0 & 1 \\
0 & 0 & 0 \\
-1 & 0 & 0
\end{array}\right), \quad A_{6}=\left(\begin{array}{ccc}
0 & -1 & 0 \\
1 & 0 & 0 \\
0 & 0 & 0
\end{array}\right)
$$

Here, we have $\sigma_{R \mathbf{x}}=R \sigma_{\mathbf{x}} R^{-1}$ and thereby $m\left(g_{1} g_{2}\right)=m\left(g_{1}\right) m\left(g_{2}\right)$ for all $g_{1}, g_{2} \in$ SE(3). Then

$$
\mathrm{d} \lambda=\lambda m\left(\gamma^{-1}\right) \mathrm{d} m(\gamma)=\lambda\left(\begin{array}{cc}
R^{-1} \mathrm{~d} R & \sigma_{R^{-1} \mathrm{~d} \mathbf{x}} \\
0 & R^{-1} \mathrm{~d} R
\end{array}\right)=\lambda\left(\begin{array}{cc}
\sigma_{\left(\omega^{4}, \ldots, \omega^{6}\right)^{T}} & \sigma_{\left(\omega^{1}, \ldots, \omega^{3}\right)^{T}} \\
0 & \sigma_{\left(\omega^{4}, \ldots, \omega^{6}\right)^{T}}
\end{array}\right),
$$

with short notation $\omega^{j}=\left.\omega^{j}\right|_{\gamma}, \lambda=\left.\lambda\right|_{\gamma}, \mathrm{d} \lambda=\left.\mathrm{d} \lambda\right|_{\gamma}$, and where we represent the covector $\lambda=\left.\sum_{i=1}^{6} \lambda_{i} \omega^{i}\right|_{\gamma}$ by a row-vector $\lambda=\left(\lambda_{1}, \ldots, \lambda_{6}\right)$. So we see that Eq. 3.10 naturally appears in Eq. 3.8: $\bar{\nabla}_{\dot{\gamma}}^{*} \lambda=0 \Leftrightarrow \frac{\mathrm{d} \lambda}{\mathrm{d} t}-\lambda m\left(\gamma^{-1}\right) \frac{\mathrm{d} m(\gamma)}{\mathrm{d} t}=0$.

Theorem 4 Let $m: \mathrm{SE}(3) \rightarrow \operatorname{Aut}\left(\mathbb{R}^{6}\right)$ denote the matrix group representation (3.10) s.t.

$$
\left.\mathrm{d} \lambda\right|_{\gamma}=\left.\lambda\right|_{\gamma} m\left(\gamma^{-1}\right) \mathrm{d} m(\gamma) \text {. }
$$

Then, along the sub-Riemannian geodesics in $\left(\mathrm{SE}(3), \Delta, \mathcal{G}_{1}\right)$, the following relation holds:

$$
\lambda(s) m(\gamma(s))^{-1}=\lambda(0) m(\gamma(0))^{-1}=\lambda(0) .
$$


Proof Note that $\left.\bar{\nabla}_{\gamma^{\prime}(s)}^{*} \lambda\right|_{\gamma(s)}=0$ iff $\left.\frac{\mathrm{d}}{\mathrm{d} s} \lambda(s)\right|_{\gamma(s)}-\left.\lambda(s)\right|_{\gamma(s)} m\left((\gamma(s))^{-1}\right) \frac{\mathrm{d}}{\mathrm{d} s} m(\gamma(s))=0$ for all $0 \leq s \leq s_{\max }(\lambda(0))$. The rest follows by

$$
\frac{\mathrm{d}}{\mathrm{d} s}\left(\lambda(s)(g(s))^{-1}\right)=-\lambda(s)(g(s))^{-1} g^{\prime}(s)(g(s))^{-1}+\lambda^{\prime}(s)(g(s))^{-1}=0,
$$

with $g(s)=m(\gamma(s))$. Multiplication with $g(s)$ from the right yields the result.

For further details, see Appendix B. These details are not necessary for the remainder of the article, where we only rely on Eqs. 3.7, 3.8, 3.10, and 3.11.

Let us recall the first integrals of the Hamiltonian system and the coadjoint orbits, that we use next for the derivation of explicit formulas for the geodesics.

Lemma 1 Co-adjoint orbits of $\lambda(0)$ are given by (see [21, p. 474] and Section 3.2)

$$
\left\{\lambda \in T^{*}(\operatorname{SE}(3)) \mid C_{1}(\lambda)=C_{1}(\lambda(0))=\mathfrak{c}^{2}, C_{2}(\lambda)=C_{2}(\lambda(0))=W\right\},
$$

where $C_{i}$ are the Casimir functions

$$
C_{1}(\lambda)=\lambda_{1}^{2}+\lambda_{2}^{2}+\lambda_{3}^{2}=\mathfrak{c}^{2}, \quad C_{2}(\lambda)=-\lambda_{1} \lambda_{4}-\lambda_{2} \lambda_{5}-\lambda_{3} \lambda_{6}=W .
$$

Corollary 2 On each co-adjoint orbit, we can choose the nice representative $\lambda(0)=$ $\left(\mathfrak{c}, 0,0,-\frac{W}{\mathfrak{c}}, 0,0\right)$, solve the Exponential map for this representative, and obtain the general solution by left-invariance. More precisely, by Theorem 4, we first find the geodesic $\tilde{\gamma}$ with

$$
\lambda(s)=\lambda(0) m(\gamma(s))=\lambda(0) m(\tilde{\gamma}(0))^{-1} m(\tilde{\gamma}(s))=\left(\mathfrak{c}, 0,0,-\frac{W}{\mathfrak{c}}, 0,0\right) m(\tilde{\gamma}(s))
$$

and then we obtain $\gamma$ via $\gamma(s)=\tilde{\gamma}^{-1}(0) \tilde{\gamma}(s)$.

\section{Sub-Riemannian Geodesics in $\mathbb{R}^{3} \rtimes S^{2}$ with Cuspless Projections}

In this section, we derive sub-Riemannian geodesics with cuspless spatial projection in the quotient $\mathbb{R}^{3} \rtimes S^{2}$, and we study geometrical properties of the geodesics, such as planarity conditions and bounds on the torsion. By Remark 5, we set $\xi=1$.

Recall that from Theorem 1, application of PMP to $\mathbf{P}_{\text {mec }}$ follows from PMP for the


horizontal part:

$$
\dot{\gamma}=\left.\lambda_{3} \mathcal{A}_{3}\right|_{\gamma}+\left.\lambda_{4} \mathcal{A}_{4}\right|_{\gamma}+\left.\lambda_{5} \mathcal{A}_{5}\right|_{\gamma},
$$

and for the vertical part, we obtain the ODE

$$
\frac{\mathrm{d}}{\mathrm{d} t}\left(\lambda_{1}, \lambda_{2}, \lambda_{3}, \lambda_{4}, \lambda_{5}\right)=\left(-\lambda_{3} \lambda_{5}, \lambda_{3} \lambda_{4}, \lambda_{1} \lambda_{5}-\lambda_{2} \lambda_{4}, \lambda_{3} \lambda_{2},-\lambda_{3} \lambda_{1}\right) .
$$

Here, $\lambda(t)=\left.\sum_{i=1}^{5} \lambda_{i}(t) \omega^{i}\right|_{\gamma(t)}$ is the momentum expressed in the moving dual frame of reference. This system of ODE's takes a simple form in $s$ parametrization, where we have $\lambda_{3}=\frac{\mathrm{d} s}{\mathrm{~d} t}>0$. It is given by

$$
\frac{\mathrm{d}}{\mathrm{d} s}\left(\lambda_{1}, \lambda_{2}, \lambda_{3}, \lambda_{4}, \lambda_{5}\right)=\left(-\lambda_{5}, \lambda_{4}, \frac{\lambda_{1} \lambda_{5}-\lambda_{2} \lambda_{4}}{\lambda_{3}}, \lambda_{2},-\lambda_{1}\right) .
$$


This system can be easily integrated as follows:

$$
\begin{array}{cc}
\lambda_{1}(s)=\lambda_{1}(0) \cosh s-\lambda_{5}(0) \sinh s, & \lambda_{5}(s)=\lambda_{5}(0) \cosh s-\lambda_{1}(0) \sinh s, \\
\lambda_{2}(s)=\lambda_{2}(0) \cosh s+\lambda_{4}(0) \sinh s, & \lambda_{4}(s)=\lambda_{4}(0) \cosh s+\lambda_{2}(0) \sinh s, \\
\lambda_{3}(s)=\sqrt{1-\left|\lambda_{4}(s)\right|^{2}-\left|\lambda_{5}(s)\right|^{2}},
\end{array}
$$

where the last expression follows from the Hamiltonian and the restriction $\lambda_{3}>0$. Thus, we obtain two hyperbolic phase portraits (see Fig. 5).

Recall that spatial arc-length parametrization is well-defined only for the geodesics $\gamma$ whose spatial projections $\pi(\gamma)=\mathbf{x}(\cdot)$ do not have external cusps. Furthermore, we evaluate $s_{\text {max }}=\min \left\{s>0 \mid u^{3}(s)=0\right\}$, i.e., the minimal positive value of $s$, such that $\pi(\gamma(s))=$ $\mathbf{x}(s)$ is a cusp point.

In the remainder of this article, we use the short notation

$$
\underline{\lambda}^{(1)}:=\left(-\lambda_{1},-\lambda_{2}\right) \text { and } \underline{\lambda}^{(2)}:=\left(\lambda_{5},-\lambda_{4}\right),
$$

which is not to be confused with the pair $\left(\lambda^{(1)}, \lambda^{(2)}\right) \in \mathbb{R}^{6}$ given by Eq. 2.7.

From the Hamiltonian $H=\frac{\lambda_{3}^{2}+\lambda_{4}^{2}+\lambda_{5}^{2}}{2}=\frac{1}{2}$, we conclude that $\left\|\underline{\lambda}^{(2)}\right\| \leq 1$. For $\left\|\underline{\lambda}^{(1)}\right\|$, we have no restrictions. This follows from $\lambda_{1}^{2}+\lambda_{2}^{2}+\lambda_{3}^{2}=\mathfrak{c}^{2}$, where $\mathfrak{c} \in \mathbb{R}$.

\subsection{Computation of the First Cusp Time}

An arbitrary geodesic in $\mathbf{P}_{\mathbf{m e c}}$ cannot be extended infinitely in $s$-parameterization, since in the common case its spatial projection presents a cusp, where spatial arclength $s$ parametrization breaks down. In fact, for any given initial values of $\underline{\lambda}^{(1)}(0)$ and $\underline{\lambda}^{(2)}(0)$, the maximum length $s_{\max }$ of such a geodesic, where we have $\kappa(s) \rightarrow \infty$ as $s \uparrow s_{\text {max }}$, is given by the following theorem.

Theorem 5 The spatial projection of a geodesic of $\mathbf{P}_{\mathbf{m e c}}$ corresponding to initial momenta $\underline{\lambda}^{(1)}(0), \underline{\lambda}^{(2)}(0)$ such that $\left\|\underline{\lambda}^{(2)}(0)\right\| \leq 1$, presents a cusp first time $t_{\text {cusp }}^{1}=t\left(s_{\text {max }}\right)$ when $s=s_{\max }$,

$$
s_{\text {max }}=\frac{1}{2} \log \frac{1+\mathfrak{c}^{2}+2 \sqrt{\mathfrak{c}^{2}-W^{2}}}{\left\|\underline{\lambda}^{(2)}(0)+\underline{\lambda}^{(1)}(0)\right\|^{2}},
$$

with $W$ and $\mathfrak{c}$ given by Lemma 1. For given $\underline{\lambda}^{(1)}(0)$ and $\underline{\lambda}^{(2)}(0)$, the spatial projection of the corresponding geodesic does not have a cusp for all end times iff $\left\|\underline{\lambda}^{(2)}(0)+\underline{\lambda}^{(1)}(0)\right\|=0$.
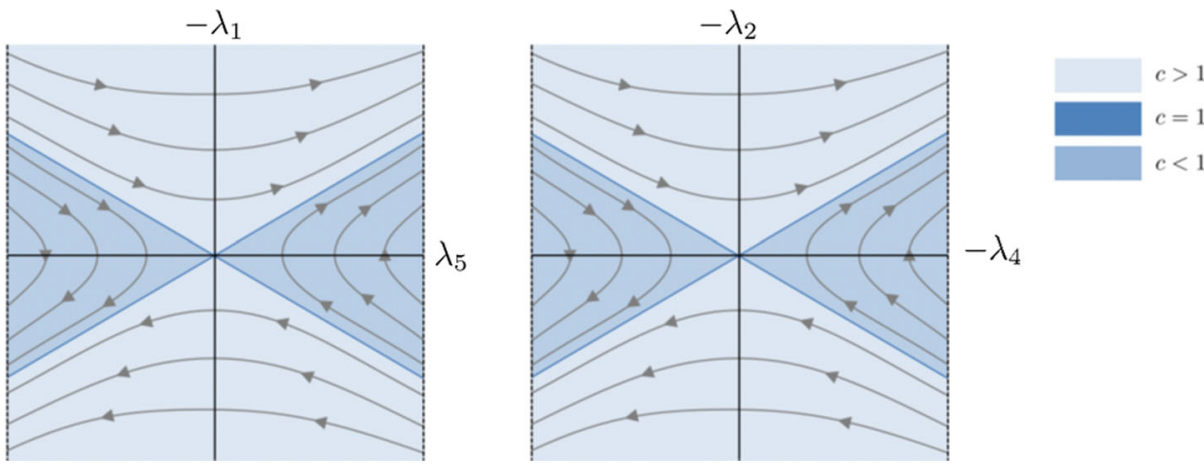

Fig. 5 Phase portraits corresponding to the components of $\underline{\lambda}^{(1)}, \underline{\lambda}^{(2)}$ satisfying the second order differential equation $\frac{\mathrm{d}^{2}}{\mathrm{~d} s^{2}} \underline{\lambda}^{(1)}(s)=\underline{\lambda}^{(1)}(s)$ along the geodesics. Several orbits are shown with arrows 
Proof By definition, we have $s_{\text {max }}=\min \left\{s>0 \mid u^{3}=0\right\}$. From PMP, we have $u^{3}(s)=$ $\lambda_{3}(s)$ for geodesics. Thus, $u^{3}(s)=0 \Leftrightarrow \lambda_{3}(s)=0$. From the Hamiltonian, we have $\lambda_{3}(s)=\sqrt{1-\left(\lambda_{4}^{2}(s)+\lambda_{5}^{2}(s)\right)}$, yielding $\lambda_{3}(s)=0 \Leftrightarrow \lambda_{4}^{2}(s)+\lambda_{5}^{2}(s)=1$. Expressions for $\lambda_{4}(s)$ and $\lambda_{5}(s)$ are given by Eq. 4.3 , so Eq. 4.5 provides the minimal positive root of $\lambda_{4}^{2}(s)+\lambda_{5}^{2}(s)=1$.

Corollary 3 For fixed $\left\|\underline{\lambda}^{(2)}(0)\right\|$ and $\left\|\underline{\lambda}^{(1)}(0)\right\|$, smax is maximal at those $\underline{\lambda}^{(2)}(0)$ and $\underline{\lambda}^{(1)}(0)$ such that $W=0$ and $\underline{\lambda}^{(2)}(0) \cdot \underline{\lambda}^{(1)}(0) \leq 0$.

Proof Let an angle $-\pi \leq \theta \leq \pi$ be chosen such that $\underline{\lambda}^{(2)}(0) \cdot \underline{\lambda}^{(1)}(0)=$ $\left\|\underline{\lambda}^{(2)}(0)\right\|\left\|\underline{\lambda}^{(1)}(0)\right\| \cos \theta$ and $\bar{W}=\left\|\underline{\lambda}^{(2)}(0)\right\|\left\|\underline{\lambda}^{(1)}(0)\right\| \sin \theta$. Then along with the condition $s_{\text {max }}>0$, Eq. 4.5 yields $\frac{\mathrm{d} s_{\max }}{\mathrm{d} \theta}=0 \Rightarrow \sin \theta=0$, yielding three critical points, $\pm \pi$ and 0 . Comparing $s_{\max }$ at these critical points, we get $\pm \pi$ as candidates for $s_{\max }$ to be maximum. Checking the second derivative at $\pm \pi$, we obtain $s_{\max }$ to be maximum at $\theta= \pm \pi$. Thus $W=0$ and $\underline{\lambda}^{(2)}(0) \cdot \underline{\lambda}^{(1)}(0)=-\left\|\underline{\lambda}^{(2)}(0)\right\|\left\|\underline{\lambda}^{(1)}(0)\right\| \leq 0$.

\subsection{The Exponential Map}

In order to integrate the geodesic equations, we apply Theorem 4. This provides the explicit formulas for the sub-Riemannian geodesics, which we present in the next theorem. The subRiemannian geodesics are parameterized by elliptic integrals of the first, second, and third kind

$$
\begin{aligned}
F(\varphi, m) & =\int_{0}^{\varphi}\left(1-m \sin ^{2} \theta\right)^{-\frac{1}{2}} \mathrm{~d} \theta, \quad E(\varphi, m)=\int_{0}^{\varphi}\left(1-m \sin ^{2} \theta\right)^{\frac{1}{2}} \mathrm{~d} \theta, \\
\Pi(n, \varphi, m) & =\int_{0}^{\varphi}\left(1-m \sin ^{2} \theta\right)^{-\frac{1}{2}}\left(1-n \sin ^{2} \theta\right)^{-1} \mathrm{~d} \theta .
\end{aligned}
$$

In our formulas below, we use constants $W=-\lambda_{2} \lambda_{5}-\lambda_{1} \lambda_{4}, \mathfrak{c}=\sqrt{\left\|\underline{\lambda}^{(1)}\right\|^{2}-\left\|\underline{\lambda}^{(2)}\right\|^{2}+1}$.

Theorem 6 Let the momentum covector be given by Eq. 4.3, where $\sum_{i=1}^{3} \lambda_{i}^{2}(0) \neq 0$. Then the spatial part of the cuspless sub-Riemannian geodesics in $\mathbf{P}_{\mathbf{m e c}}$ is given by

$$
\mathbf{x}(s)=\tilde{R}(0)^{T}(\tilde{\mathbf{x}}(s)-\tilde{\mathbf{x}}(0)),
$$

where $\tilde{R}(0)$ and $\tilde{\mathbf{x}}(s):=(\tilde{x}(s), \tilde{y}(s), \tilde{z}(s))$ are given in terms of $\underline{\lambda}^{(1)}(0)$ and $\underline{\lambda}^{(2)}(0)$ depending on several cases. For all cases with $\underline{\lambda}^{(1)}(0) \neq \underline{\lambda}^{(2)}(0)$, we have

$$
\tilde{x}(s)=\frac{1}{\mathfrak{c}} \int_{0}^{s} \lambda_{3}(\tau) \mathrm{d} \tau=-\frac{i \sqrt{1-d} \sqrt{1+\mathfrak{c}^{2}}}{\mathfrak{c} \sqrt{2}}\left(E\left(\left(s+\frac{\varphi}{2}\right) i, M\right)-E\left(\frac{\varphi}{2} i, M\right)\right),
$$

where $M:=\frac{2 d}{d-1}, d:=\frac{\left\|\underline{\lambda}(2)(0)+\underline{\lambda}^{(1)}(0)\right\|\|\| \underline{\lambda}^{(2)}(0)-\underline{\lambda}^{(1)}(0) \|}{1+\mathfrak{c}^{2}} \leq 1$, and $\varphi:=\log \frac{\left\|\underline{\lambda}^{(2)}(0)+\lambda^{(1)}(0)\right\|}{\left\|\underline{\lambda}^{(2)}(0)-\underline{\lambda}^{(1)}(0)\right\|}$.

For the case $\underline{\lambda}^{(1)}(0)=\mathbf{0}$, we have

$$
\tilde{R}(0)=\left(\begin{array}{ccc}
0 & 0 & 1 \\
0 & 1 & 0 \\
-1 & 0 & 0
\end{array}\right) \in \operatorname{SO}(3), \quad\left(\begin{array}{c}
\tilde{y}(s) \\
\tilde{z}(s)
\end{array}\right)=\frac{-1}{\mathfrak{c}}\left(\begin{array}{c}
\lambda_{4}(s) \\
\lambda_{5}(s)
\end{array}\right) .
$$


For the case $\underline{\lambda}^{(1)}(0) \neq \mathbf{0}$, we have

$$
\tilde{R}(0)=\frac{1}{\mathfrak{c}}\left(\begin{array}{ccc}
\lambda_{1}(0) & \lambda_{2}(0) & \lambda_{3}(0) \\
\mathfrak{c} \frac{-\lambda_{2}(0)}{\left\|\lambda^{(1)}(0)\right\|} & \mathfrak{c} \frac{\lambda_{1}(0)}{\left\|\lambda^{(1)}(0)\right\|} & 0 \\
\frac{-\lambda_{1}^{(0)}(0) \lambda_{3}(0)}{\left\|\underline{\lambda}^{(1)}(0)\right\|} & \frac{-\lambda_{2}^{(2}(0) \lambda_{3}(0)}{\left\|\underline{\lambda}^{(1)}(0)\right\|} & \left\|\underline{\lambda}^{(1)}(0)\right\|
\end{array}\right) \in \operatorname{SO}(3) .
$$

For the case $W=0$ along with $\underline{\lambda}^{(1)}(0) \neq \mathbf{0}$, we have

$$
\left(\begin{array}{c}
\tilde{y}(s) \\
\tilde{z}(s)
\end{array}\right)=\frac{\underline{\lambda}^{(2)}(s) \cdot \underline{\lambda}^{(1)}(0)}{\mathfrak{c}\left\|\underline{\lambda}^{(1)}(0)\right\|}\left(\begin{array}{l}
0 \\
1
\end{array}\right) .
$$

For $W \neq 0$ along with $\underline{\lambda}^{(1)}(0) \neq \mathbf{0}$, we have

$$
\left(\begin{array}{c}
\tilde{y}(s) \\
\tilde{z}(s)
\end{array}\right)=\frac{\sqrt{\left\|\underline{\lambda}^{(2)}(s)\right\|^{2}-W^{2} \mathfrak{c}^{-2}}}{\mathfrak{c}^{2}\left\|\underline{\lambda}^{(1)}(0)\right\| \sqrt{\left\|\underline{\lambda}^{(2)}(0)\right\|^{2}-W^{2} \mathfrak{c}^{-2}}}\left(\begin{array}{cc}
\cos \tilde{\psi}(s) & -\sin \tilde{\psi}(s) \\
\sin \tilde{\psi}(s) & \cos \tilde{\psi}(s)
\end{array}\right)\left(\begin{array}{c}
W \lambda_{3}(0) \\
\mathfrak{c}\left(\underline{\lambda}^{(2)}(0) \cdot \underline{\lambda}^{(1)}(0)\right)
\end{array}\right),
$$

where

$$
\begin{aligned}
\tilde{\psi}(s)= & \int_{0}^{s} \frac{W \mathfrak{c}^{-1} \lambda_{3}(\tau)}{\left\|\underline{\lambda}^{(2)}(\tau)\right\|^{2}-W^{2} \mathfrak{c}^{-2}} \mathrm{~d} \tau=-\frac{W}{\mathfrak{c}} \frac{\sqrt{2}}{\sqrt{1+\mathfrak{c}^{2}} \sqrt{1-d}} \frac{1}{i}\left(F\left(i\left(s+\frac{\varphi}{2}\right), M\right)-F\left(\frac{i \varphi}{2}, M\right)\right. \\
& \left.-\left(1-\frac{1}{D}\right)\left(\Pi\left(\frac{M}{D}, i\left(s+\frac{\varphi}{2}\right), M\right)-\Pi\left(\frac{M}{D}, \frac{i \varphi}{2}, M\right)\right)\right),
\end{aligned}
$$

with $D=2\left(\frac{W^{2}}{\mathfrak{c}^{2}}-1\right)\left(1+\mathfrak{c}^{2}\right)^{-1}(1-d)^{-1}+1$ and $|\tilde{\psi}(s)|<\pi, \operatorname{sign}(\tilde{\psi}(s))=\operatorname{sign}(W)$.

Proof We use Theorem 4 and apply Corollary 2. From which we have $\gamma(s)=\tilde{\gamma}(0)^{-1} \tilde{\gamma}(s)$, where $m(\tilde{\gamma}(s))$ relates to $\lambda(s)$ via Eq. 3.13. This provides Eq. 4.6. For the most general case, assuming non-vanishing denominators throughout, we see that when choosing (4.9) and $\tilde{\mathbf{x}}(0):=\frac{1}{\mathfrak{c}^{2}\left\|\underline{\lambda}^{(1)}(0)\right\|}\left(0, W \lambda_{3}(0), \mathfrak{c}\left(\underline{\lambda}^{(1)}(0) \cdot \underline{\lambda}^{(2)}(0)\right)\right)^{T}$, Eq. 3.13 is satisfied in the initial moment $s=0$. Then solving Eq. 3.13 for $\tilde{x}, \tilde{y}$, and $\tilde{z}$, we obtain $\tilde{x}(s)=\frac{1}{\mathfrak{c}} \int_{0}^{s} \lambda_{3}(\tau) \mathrm{d} \tau$ for $\tilde{x}(s)$ and for $(\tilde{y}(s), \tilde{z}(s))$ we obtain the following system:

$$
\begin{aligned}
& \left(\begin{array}{l}
\tilde{y}^{\prime}(s) \\
\tilde{z}^{\prime}(s)
\end{array}\right)=A(s)\left(\begin{array}{c}
\tilde{y}(s) \\
\tilde{z}(s)
\end{array}\right), \\
& \text { with } A(s)=\frac{1}{\left\|\underline{\lambda}^{(2)}(0)\right\|^{2}-\frac{W^{2}}{c^{2}}}\left(\begin{array}{cc}
\underline{\lambda}^{(2)}(s) \cdot \underline{\lambda}^{(1)}(s) & -\frac{W}{\mathfrak{c}} \lambda_{3}(s) \\
\frac{W}{c} \lambda_{3}(s) & \underline{\lambda}^{(2)}(s) \cdot \underline{\lambda}^{(1)}(s)
\end{array}\right) .
\end{aligned}
$$

Note that $A(s)$ and $A\left(s^{\prime}\right)$ commute, and Wilcox formula [37] yields the result.

Clearly, the formulas are not valid when denominators vanish. Hence, we do the whole procedure keeping in mind the special cases (4.8), (4.10) right from the start and get the required results.

Regarding Eq. 4.11, we note that matrix $e^{\int_{0}^{s} A(\tau) \mathrm{d} \tau}$ can be computed explicitly. One has




with $\tilde{\psi}(s)=\int_{0}^{s} \frac{W \mathfrak{c}^{-1} \lambda_{3}(\tau)}{\left\|\underline{\lambda}^{(2)}\left(s^{\prime}\right)\right\|^{2}-W^{2} \mathfrak{c}^{-2}} \mathrm{~d} \tau$. From the first integrals, one can deduce that $\forall s \in$ $\left(0, s_{\max }\right):\left\|\underline{\lambda}^{(2)}(s)\right\|^{2}-W^{2} \mathfrak{c}^{-2}>0$. Note $\left\|\underline{\lambda}^{(2)}(s)\right\|^{2}=W^{2} \mathfrak{c}^{-2} \Leftrightarrow s=s_{\max } \wedge \underline{\lambda}^{(1)}\left(s_{\max }\right)$. $\underline{\lambda}^{(2)}\left(s_{\max }\right)=0$. By direct computation, (4.12) follows. Moreover, by Lemma 4.13 in [16], we have $|\tilde{\psi}(s)|<\pi$ for all $s \leq s_{\max }$ and since $\frac{\mathfrak{c}^{-1} \lambda_{3}(s)}{\left\|\underline{\lambda}^{(2)}(s)\right\|^{2}-W^{2} \mathfrak{c}^{-2}} \geq 0$ we have $\operatorname{sign}(\tilde{\psi}(s))=$ $\operatorname{sign}(W)$.

The remaining part is to prove that $\tilde{x}(s)=\frac{1}{\mathfrak{c}} \int_{0}^{s} \lambda_{3}(\tau) \mathrm{d} \tau$ can be integrated in terms of elliptic integrals, as presented in Eq. 4.7. This is done by the following computation:

$\tilde{x}(s)=\int_{0}^{s} \frac{\lambda_{3}(\tau)}{\mathfrak{c}} \mathrm{d} \tau=\frac{1}{\mathfrak{c}} \int_{0}^{s} \sqrt{1-\left\|\underline{\lambda}^{(2)}(\tau)\right\|^{2}} \mathrm{~d} \tau=\frac{\sqrt{1+\mathfrak{c}^{2}}}{\mathfrak{c} \sqrt{2}} \int_{0}^{s} \sqrt{1-c_{1} \cosh (2 \tau)-c_{2} \sinh (2 \tau)} \mathrm{d} \tau$,

where $c_{1}:=\frac{1}{1+\mathfrak{c}^{2}}\left(\left\|\underline{\lambda}^{(2)}(0)\right\|^{2}+\left\|\underline{\lambda}^{(1)}(0)\right\|^{2}\right)$ and $c_{2}:=\frac{2}{1+\mathfrak{c}^{2}} \underline{\lambda}^{(2)}(0) \cdot \underline{\lambda}^{(1)}(0)$.

Denoting $d:=\sqrt{c_{1}^{2}-c_{2}^{2}}, \varphi:=\frac{1}{2} \log \frac{c_{1}+c_{2}}{c_{1}-c_{2}}, M:=\frac{2 d}{d-1}$ and $\theta:=i \tau+\frac{i \varphi}{2}$, we can express

$$
\begin{aligned}
\tilde{x}(s) & =\frac{\sqrt{1+\mathfrak{c}^{2}}}{\mathfrak{c} \sqrt{2}} \int_{0}^{s} \sqrt{1-d \cos (2 i \tau+i \varphi)} \mathrm{d} \tau=\frac{\sqrt{1-d} \sqrt{1+\mathfrak{c}^{2}}}{i \mathfrak{c} \sqrt{2}} \int_{\frac{i \varphi}{2}}^{i\left(s+\frac{\varphi}{2}\right)} \sqrt{1-M \sin ^{2}(\theta)} \mathrm{d} \theta \\
& =-\frac{i \sqrt{1-d} \sqrt{1+\mathfrak{c}^{2}}}{\mathfrak{c} \sqrt{2}}\left(E\left(\left(s+\frac{\varphi}{2}\right) i, M\right)-E\left(\left(\frac{\varphi}{2}\right) i, M\right)\right),
\end{aligned}
$$

where we note that $d \leq \frac{1}{1+\mathfrak{c}^{2}} \frac{1}{2}\left(\left\|\underline{\lambda}^{(2)}(0)-\underline{\lambda}^{(1)}(0)\right\|^{2}+\left\|\underline{\lambda}^{(2)}(0)+\underline{\lambda}^{(1)}(0)\right\|^{2}\right) \leq 1$.

Corollary 4 The exponential map Exp : $\mathcal{D}_{0} \rightarrow \mathbb{R}^{3} \rtimes S^{2}$ defined in Definition 6 is given by

$$
\operatorname{Exp}(\lambda(0), L)=\left(\mathbf{x}^{*}(L), \mathbf{n}^{*}(L)\right),
$$

where $\mathbf{n}^{*}(L)=\left.\frac{d}{d s} \mathbf{x}^{*}(s)\right|_{s=L}$ and where the spatial part of the geodesic $\mathbf{x}^{*}(L)=$ $\left(x^{*}(L), y^{*}(L), z^{*}(L)\right)$ is explicitly given by Eq. 4.6. Here, the tangent equals

$$
\left.\frac{d}{d s} \mathbf{x}^{*}(s)\right|_{s=L}=\mathbf{x}^{*^{\prime}}(L)=(\tilde{R}(0))^{T} \tilde{\mathbf{x}}^{\prime}(L),
$$

with $\tilde{R}(0)$ given by Eqs. 4.8 and 4.9, and $\tilde{x}^{\prime}(L)=\frac{1}{\mathfrak{c}} \lambda_{3}(L)$ with $\lambda_{3}(L)$ given by Eq. 4.3 , and $\left(\tilde{y}^{\prime}(L), \tilde{z}^{\prime}(L)\right)^{T}=A(L)(\tilde{y}(L), \tilde{z}(L))^{T}$ with $A(L)$ given by Eq. 4.13 and $\tilde{y}(L), \tilde{z}(L)$ given by Eq. 4.11 .

\subsection{Geometric Properties of the Stationary Curves}

Let a stationary curve of $\mathbf{P}_{\text {curve }}$ in $\mathbb{R}^{3}$ be given by $\mathbf{x}:[0, L] \rightarrow \mathbb{R}^{3}$ parameterized by arclength denoted by $s$. Let the unit tangent, the unit normal, and the unit binormal for this curve be given by $\mathbf{T}, \mathbf{N}$, and $\mathbf{B}$, respectively. Let $\kappa$ and $\tau$ denote curvature and torsion, then the Frenet-Serret equations are

$$
\frac{\mathrm{d}}{\mathrm{d} s}\left(\begin{array}{l}
\mathbf{T}(s) \\
\mathbf{N}(s) \\
\mathbf{B}(s)
\end{array}\right)=\left(\begin{array}{ccc}
0 & \kappa(s) & 0 \\
-\kappa(s) & 0 & \tau(s) \\
0 & -\tau(s) & 0
\end{array}\right)\left(\begin{array}{l}
\mathbf{T}(s) \\
\mathbf{N}(s) \\
\mathbf{B}(s)
\end{array}\right)
$$


Let us first study the curvature and signed torsion of the spatial projection of the subRiemannian geodesics. Let us recall the first integral constants $W=-\lambda_{2} \lambda_{5}-\lambda_{1} \lambda_{4}$ and $\mathfrak{c}=\sqrt{\left\|\underline{\lambda}^{(1)}\right\|^{2}-\left\|\underline{\lambda}^{(2)}\right\|^{2}+1}$ in Lemma 1. Furthermore, we have that

$$
R^{\prime}=\frac{d t}{d s} \dot{R}=\lambda_{3}^{-1} \dot{R}=\lambda_{3}^{-1} R\left(\begin{array}{ccc}
0 & 0 & \lambda_{5} \\
0 & 0 & -\lambda_{4} \\
-\lambda_{5} & \lambda_{4} & 0
\end{array}\right) \text {. }
$$

and therefore

$$
\mathbf{x}^{\prime}(s)=R(s) \mathbf{e}_{z} \Rightarrow \mathbf{x}^{\prime \prime}(s)=R(s)\left(\begin{array}{c}
\frac{\lambda_{5}(s)}{\lambda_{3}(s)} \\
-\frac{\lambda_{4}(s)}{\lambda_{3}(s)} \\
0
\end{array}\right) \Rightarrow \mathbf{x}^{\prime \prime \prime}(s)=R(s)\left(\begin{array}{c}
\frac{d}{d s}\left(\frac{\lambda_{5}(s)}{\lambda_{3}(s)}\right) \\
-\frac{d}{d s}\left(\frac{\lambda_{4}(s)}{\lambda_{3}(s)}\right) \\
-\frac{\lambda_{4}^{2}(s)+\lambda_{5}^{2}(s)}{\lambda_{3}^{2}(s)}
\end{array}\right) .
$$

Theorem 7 The absolute curvature and the signed torsion of a stationary curve of $\mathbf{P}_{\text {curve }}$ are given by

$$
\kappa=\frac{\sqrt{\lambda_{4}^{2}+\lambda_{5}^{2}}}{\lambda_{3}}=\frac{\sqrt{1-\lambda_{3}^{2}}}{\lambda_{3}}, \quad \tau=\frac{W}{\lambda_{4}^{2}+\lambda_{5}^{2}},
$$

with momentum components $\lambda_{i}$ given by Eq. 4.3. We have the following fundamental relation between curvature and torsion

$$
\tau(s) \kappa^{2}(s)=W\left(t^{\prime}(s)\right)^{2} .
$$

The torsion is bounded as follows

$$
|W| \leq|\tau(s)| \leq \frac{2|W|}{\sqrt{\left(1-\mathfrak{c}^{2}\right)^{2}+4 W^{2}}+1-\mathfrak{c}^{2}} \text { for all } 0 \leq s \leq L \leq s_{\max } .
$$

Proof In the proof, we use the following properties of norm, inner product, and cross product:

$$
\forall_{R \in \mathrm{SO}(3)} \forall_{\mathbf{a}, \mathbf{b} \in \mathbb{R}^{3}}:\|R \mathbf{a}\|=\|\mathbf{a}\|, \quad(R \mathbf{a}) \cdot(R \mathbf{b})=\mathbf{a} \cdot \mathbf{b}, \quad(R \mathbf{a}) \times(R \mathbf{b})=R(\mathbf{a} \times \mathbf{b}) .
$$

First part follows by straightforward computation via Eqs. 4.15 and 4.16. By definition, we have $\kappa(s)=\left\|\mathbf{x}^{\prime \prime}(s)\right\|$. Thus by Eq. 4.16 and the Hamiltonian $H=\frac{1}{2}\left(\lambda_{3}^{2}+\lambda_{4}^{2}+\lambda_{5}^{2}\right)=\frac{1}{2}$, we obtain, that the curvature satisfies (4.17). For arclength parametrized curve $\mathbf{x}(s)$ in $\mathbb{R}^{3}$, the torsion is given by $\tau=\frac{\left(\mathbf{x}^{\prime} \times \mathbf{x}^{\prime \prime}\right) \cdot \mathbf{x}^{\prime \prime \prime}}{\left\|\mathbf{x}^{\prime} \times \mathbf{x}^{\prime \prime}\right\|^{2}}$ (see e.g. [35]). Thus by Eq. 4.16, we have $\left(\mathbf{x}^{\prime} \times \mathbf{x}^{\prime \prime}\right) \cdot \mathbf{x}^{\prime \prime \prime}=$ $\frac{W}{\lambda_{3}^{2}}$ and $\left\|\mathbf{x}^{\prime} \times \mathbf{x}^{\prime \prime}\right\|^{2}=\frac{\lambda_{4}^{2}+\lambda_{5}^{2}}{\lambda_{3}^{2}}$, and thereby $\tau$ satisfies (4.17). Equation 4.18 follows by $\lambda_{3}=\frac{d s}{d t}$ and Eq. 4.17.

In order to prove bounds (4.19), we use expression for torsion $\tau=\frac{W}{\lambda_{4}^{2}+\lambda_{5}^{2}} \Rightarrow|\tau|=$ $\frac{|W|}{\lambda_{4}^{2}+\lambda_{5}^{2}}$. The lower bound in Eq. 4.19 holds since $\lambda_{4}^{2}+\lambda_{5}^{2} \leq 1$ due to the Hamiltonian.

To prove the upper bound, we show that $\lambda_{4}^{2}(s)+\lambda_{5}^{2}(s) \geq$ $\frac{1}{2}\left(\sqrt{\left(1-\mathfrak{c}^{2}\right)^{2}+4 W^{2}}+1-\mathfrak{c}^{2}\right)$ for all $s \in\left[0, s_{\max }\right]$. To obtain the last inequality, we solve $\frac{\mathrm{d}\left(\lambda_{4}^{2}(s)+\lambda_{5}^{2}(s)\right)}{\mathrm{d} s}=0$ using Eq. 4.3:

$s_{M}:=\arg \min _{s \in\left[0, s_{\text {max }}\right]}\left\{\lambda_{4}^{2}(s)+\lambda_{5}^{2}(s)\right\}=\left\{\begin{array}{l}0, \text { if }-\lambda_{5}(0) \lambda_{1}(0)+\lambda_{2}(0) \lambda_{4}(0)>0, \\ \frac{1}{2} \log \frac{\left\|\lambda^{(2)}(0)-\lambda^{(1)}(0)\right\|}{\left\|\underline{\lambda}^{(2)}(0)+\underline{\lambda}^{(1)}(0)\right\|}, \text { if }-\lambda_{5}(0) \lambda_{1}(0)+\lambda_{2}(0) \lambda_{4}(0) \leq 0 .\end{array}\right.$ 
Thus, evaluation $\left|\tau\left(s_{M}\right)\right|=\frac{|W|}{\lambda_{4}^{2}\left(s_{M}\right)+\lambda_{5}^{2}\left(s_{M}\right)}$ gives the upper bound in Eq. 4.19. Here, we use identity $\left\|\underline{\lambda}^{(2)}(0)+\underline{\lambda}^{(1)}(0)\right\|\left\|\underline{\lambda}^{(2)}(0)-\underline{\lambda}^{(1)}(0)\right\|=\sqrt{\left(1-\mathfrak{c}^{2}\right)^{2}+4 W^{2}}$.

Corollary 5 The cuspless spatial projections of sub-Riemannian geodesics of $\mathbf{P}_{\mathbf{m e c}}$ with $\sum_{i=1}^{3} \lambda_{i}^{2}(0) \neq 0$ (i.e., the stationary curves of $\mathbf{P}_{\text {curve }}$ ) are planar if and only if $W=0$.

Next, we show that when taking the end conditions to be co-planar, one gets $W=0$ implying that planar curves are the only cuspless geodesic of problem $\mathbf{P}_{\text {mec }}$ connecting these conditions.

Theorem $\mathbf{8}$ Let $\mathbf{x}:\left[0, s_{\max }\right] \rightarrow \mathbb{R}^{3}$ be the spatial part of a cuspless sub-Riemannian geodesic of $\mathbf{P}_{\mathbf{m e c}}$ given by Theorem 6, i.e., let $\mathbf{x}$ be a stationary curve of $\mathbf{P}_{\text {curve. Then for any }}$ $s \in\left(0, s_{\text {max }}\right]$, one has that $\mathbf{e}_{z}, \mathbf{x}(s)$ and $\mathbf{x}^{\prime}(s)$ are coplanar if and only if $\mathbf{x}$ is a planar curve, i.e.

$$
\mathbf{e}_{z} \cdot\left(\mathbf{x}(s) \times \mathbf{x}^{\prime}(s)\right)=0 \Leftrightarrow W=0 .
$$

Proof If $W=0$, it follows by Corollary 5 that the curve is planar. By Theorem 6 , we indeed get that $W=0 \Rightarrow \tilde{y}(s) \equiv 0$ and $\tilde{y}(s) \equiv 0 \Rightarrow \tilde{\mathbf{x}}(0) \cdot\left(\tilde{\mathbf{x}}(s) \times \tilde{\mathbf{x}}^{\prime}(s)\right)=0 \Rightarrow \mathbf{e}_{z} \cdot\left(\mathbf{x}(s) \times \mathbf{x}^{\prime}(s)\right)=$ 0 .

Now, we focus on the other direction of the implication. Let us consider curve $\mathbf{x}^{\prime}$ : $\left[0, s_{\text {max }}\right] \rightarrow S^{2}$. It can have a minimum curvature of 1 if it aligns with a great circle on $S^{2}$. By Eqs. 4.16 and 4.17, it follows that the geodesic curvature $\mathcal{K}_{\text {tan }}$ of $\mathbf{x}^{\prime}(\cdot)$ is given as

$$
\mathcal{K}_{\text {tan }}(s):=\frac{\left\|\mathbf{x}^{\prime \prime}(s) \times \mathbf{x}^{\prime \prime \prime}(s)\right\|}{\left\|\mathbf{x}^{\prime \prime}(s)\right\|^{3}}=\frac{\sqrt{\kappa^{6}(s)+\left(\kappa^{2}(s)+1\right)^{2} W^{2}}}{\kappa^{3}(s)}=\sqrt{1+\frac{\left(\kappa^{2}(s)+1\right)^{2} W^{2}}{\kappa^{6}(s)}} .
$$

Thus, $W \neq 0 \Rightarrow \mathcal{K}_{\text {tan }}(s)>1$ for any $s \in\left(0, s_{\text {max }}\right)$. The curve $\mathbf{x}^{\prime}$ gets aligned with great circles only at cusp points where $\kappa(s)=\infty$ which never occurs in an interior point. Thus the curve $\mathbf{n}=\mathbf{x}^{\prime}$ can intersect a great circle on $S^{2}$ at most at two points. Therefore, any three points along this curve can never lie simultaneously on a plane passing through the origin. Thus

$\forall_{\tau, s>0}: \mathbf{x}^{\prime}(0) \cdot\left(\mathbf{x}^{\prime}(\tau) \times \mathbf{x}^{\prime}(s)\right) \neq 0 \Rightarrow \int_{0}^{s}\left(\mathbf{x}^{\prime}(0) \cdot\left(\mathbf{x}^{\prime}(\tau) \times \mathbf{x}^{\prime}(s)\right)\right) \mathrm{d} \tau \neq 0 \Rightarrow \mathbf{x}^{\prime}(0) \cdot\left(\mathbf{x}(s) \times \mathbf{x}^{\prime}(s)\right) \neq 0$.

So $W \neq 0 \Rightarrow \mathbf{e}_{z} \cdot\left(\mathbf{x}(s) \times \mathbf{x}^{\prime}(s)\right) \neq 0$. In turn, this leads to $\mathbf{e}_{z} \cdot\left(\mathbf{x}(s) \times \mathbf{x}^{\prime}(s)\right)=0 \Rightarrow$ $W=0$.

The following corollaries relate the planar cuspless sub-Riemannian geodesics in $\left(\operatorname{SE}(3), \Delta, \mathcal{G}_{1}\right)$ to those in $\left(\operatorname{SE}(2), \tilde{\Delta}, \tilde{\mathcal{G}}_{1}\right)$ with $\tilde{\Delta}=\operatorname{ker}\{-\sin \theta \mathrm{d} x+\cos \theta \mathrm{d} y\}$ and $\tilde{\mathcal{G}}_{1}=$ $(\cos \theta \mathrm{d} x+\sin \theta \mathrm{d} y) \otimes(\cos \theta \mathrm{d} x+\sin \theta \mathrm{d} y)+\mathrm{d} \theta \otimes \mathrm{d} \theta)$, cf. [11].

Corollary 6 Given admissible coplanar end conditions for $\mathbf{P}_{\text {curve, the unique cuspless }}$ stationary curve connecting them is planar.

Now by the global optimality results in $[6,11]$, we have the following corollary. 
Corollary 7 Let $\tilde{\mathcal{R}}$ denote the range of the exponential map of cuspless geodesics in $S E(2)$, which coincides with the set of admissible end conditions of $\mathbf{P}_{\text {curve }}$ in $\mathrm{SE}(2)$. Then the set $\left\{\left(\mathbf{x}_{1},\left(\frac{x_{1}}{\sqrt{x_{1}^{2}+y_{1}^{2}}} \sin \theta_{1}, \frac{y_{1}}{\sqrt{x_{1}^{2}+y_{1}^{2}}} \sin \theta_{1}, \cos \theta_{1}\right)^{T}\right) \mid\left(\mathbf{x}_{1}, \theta_{1}\right) \in \tilde{\mathcal{R}}\right\} \subset \mathcal{R}$ (recall (2.11)) is a set of end conditions admitting a unique global cuspless minimizer of $\mathbf{P}_{\text {curve. }}$

Next, we show that the sub-Riemannian geodesics do not self intersect or roll up, despite the fact that the absolute curvature $\kappa(s) \rightarrow \infty$ as $s \uparrow s_{\text {max }}$.

Corollary 8 The cuspless spatial projections of sub-Riemannian geodesics of $\mathbf{P}_{\mathbf{m e c}}$ with $\lambda_{1}^{2}(0)+\lambda_{2}^{2}(0)+\lambda_{3}^{2}(0) \neq 0$, have a monotonically increasing component along $\mathfrak{c}^{-1}\left(\lambda_{1}(0) \mathbf{e}_{x}+\lambda_{2}(0) \mathbf{e}_{y}+\lambda_{3}(0) \mathbf{e}_{z}\right)$. Hence, they do not self intersect or roll up.

Proof From Eq. 4.6, we have $\mathbf{e}_{\tilde{x}}=(\tilde{R}(0))^{T} \mathbf{e}_{x}=\mathfrak{c}^{-1}\left(\lambda_{1}(0) \mathbf{e}_{x}+\lambda_{2}(0) \mathbf{e}_{y}+\lambda_{3}(0) \mathbf{e}_{z}\right)$. By Theorem 6 , we have $\tilde{x}^{\prime}(s)>0$ for all $s \in\left(0, s_{\text {max }}\right)$ and the result follows.

Next, we want to study bounds on the set $\mathcal{R}$, recall (2.11). Our numerical investigations clearly show that the spatial part of all points in $\mathcal{R}$ is contained in the half space $z \geq 0$, and that the plane $z=0$ is only reached with $U$-shaped planar geodesics (i.e., $W=0, \mathfrak{c}<1$ ) at $s=s_{\max }$. These numerical observations inspired us to find the natural generalization of formal results in [11, Thm.7\&8] on the SE(2)-case. We partly succeeded as we show in the next three corollaries, which provide bounds on the set $\mathcal{R}$.

Corollary 9 Let $s \mapsto \gamma(s)=(x(s), y(s), z(s), R(s))$ be a sub-Riemannian geodesic in $\left(\mathrm{SE}(3), \Delta, \mathcal{G}_{1}\right)$ with $\lambda_{6}=0$ and $\lambda_{1}^{2}(0)+\lambda_{2}^{2}(0)+\lambda_{3}^{2}(0) \neq 0$, departing from $e=(\mathbf{0}$, I), s.t. the spatial projection is cuspless.

$$
\begin{aligned}
& \text { If } \underline{\lambda}^{(1)}(0) \cdot \underline{\lambda}^{(2)}(0) \geq 0 \text {, then } z(s)>0 \text { for all } s \in\left(0, s_{\text {max }}\right) . \\
& \text { If } \underline{\lambda}^{(1)}(0) \cdot \underline{\lambda}^{(2)}(0)<0 \text {, then } z(s)>0 \text { for all } s \in\left(0, s_{m}\right) \text {, with }
\end{aligned}
$$

$$
s_{m}=\left\{\begin{array}{l}
\log \frac{\left|\lambda_{4}(0)+\lambda_{5}(0)\right|}{\left|\lambda \lambda_{4}(0)-\lambda_{5}(0)\right|}, \text { if } \underline{\lambda}^{(1)}(0)=-\underline{\lambda}^{(2)}(0), \\
\log \frac{\left\|\underline{\lambda}^{(2)}(0)+\underline{\lambda}^{(1)}(0)\right\|}{\left\|\underline{\lambda}^{(2)}(0)-\underline{\lambda}^{(1)}(0)\right\|}, \text { otherwise. }
\end{array}\right.
$$

Proof If $\underline{\lambda}^{(1)}(0) \cdot \underline{\lambda}^{(2)}(0) \geq 0$, then $\underline{\lambda}^{(1)}(\tau) \cdot \underline{\lambda}^{(2)}(\tau) \geq 0$ for all $\tau \in\left(0, s_{\text {max }}\right)$, which implies $A(\tau) \geq 0$ for all $\tau \in\left(0, s_{\max }\right)$, where we recall Eq. 4 .13. Now by Theorem 6 , we have

$$
\begin{aligned}
& z(s)=\left(\mathbf{x}(s), \mathbf{e}_{z}\right)=\left((\tilde{R}(0))^{T}(\tilde{\mathbf{x}}(s)-\tilde{\mathbf{x}}(0)), \mathbf{e}_{z}\right)=\left(\tilde{\mathbf{x}}(s)-\tilde{\mathbf{x}}(0), \tilde{R}(0) \mathbf{e}_{z}\right)=\left(\tilde{\mathbf{x}}(s)-\tilde{\mathbf{x}}(0), \tilde{\mathbf{x}}^{\prime}(0)\right)
\end{aligned}
$$



$$
\begin{aligned}
& =\mathfrak{c}^{-2} \lambda_{3}(s) \int_{0}^{s} \lambda_{3}(\tau) \mathrm{d} \tau+\left((A(0))^{T}\left(e^{0} A(\tau) \mathrm{d} \tau-I\right)\left(\begin{array}{c}
\tilde{y}(0) \\
\tilde{z}(0)
\end{array}\right),\left(\begin{array}{c}
\tilde{y}(0) \\
\tilde{z}(0)
\end{array}\right)\right) .
\end{aligned}
$$

As cusps do not occur on the interior of spatially projected curve $\mathbf{x}(\cdot)=(x(\cdot), y(\cdot), z(\cdot))$, the first term $\mathfrak{c}^{-2} \lambda_{3}(s) \int_{0}^{s} \lambda_{3}(\tau) \mathrm{d} \tau$ is strictly positive for all $s \in\left(0, s_{\text {max }}\right)$. Regarding the 
second term, we note that $A\left(\tau_{1}\right)$ and $A\left(\tau_{2}\right)$ commute for all $\tau_{1}, \tau_{2}>0$ and each $A(\tau)$ can be diagonalized. Now, $A^{T}(0)=C A^{-1}(0)$ for some $C>0$. Thereby, both operators $A^{T}(0)$ and $\left(e^{\int_{0}^{s} A(\tau) \mathrm{d} \tau}-I\right)$ commute, have a common eigensystem, and are either semi-positive definite or semi-negative definite, thus

$$
\begin{aligned}
& z(s)>\left((A(0))^{T}\left(e^{\int} A(\tau) \mathrm{d} \tau-I\right)\left(\begin{array}{c}
\tilde{y}(0) \\
\tilde{z}(0)
\end{array}\right),\left(\begin{array}{c}
\tilde{y}(0) \\
\tilde{z}(0)
\end{array}\right)\right) \geq 0 \Leftrightarrow
\end{aligned}
$$

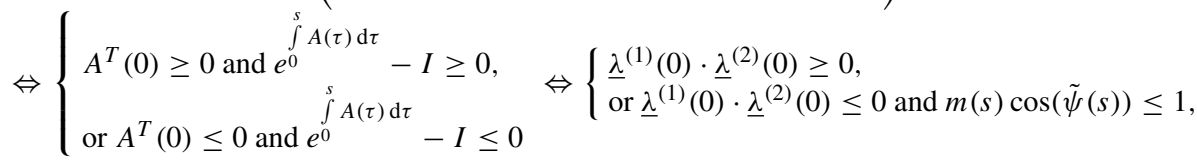

where the scalar multiplier

$$
m(s)=\sqrt{\frac{1-\left|\lambda_{3}(s)\right|^{2}-W^{2} \mathfrak{c}^{-2}}{1-\left|\lambda_{3}(0)\right|^{2}-W^{2} \mathfrak{c}^{-2}}} \leq 1 \Leftrightarrow \lambda_{3}(s) \geq \lambda_{3}(0),
$$

comes from Eq. 4.13. Now, $s_{m}$ is chosen as the first positive root of $\lambda_{3}(s)=\lambda_{3}(0)$ and the result follows as $m(s) \leq 1 \Rightarrow m(s) \cos (\tilde{\psi}(s))-1 \leq 0$.

Corollary 10 Let $W=0$ then all cuspless sub-Riemannian geodesics in $\left(\mathrm{SE}(3), \Delta, \mathcal{G}_{1}\right)$ with $\lambda_{6}=0$ and $\lambda_{1}^{2}(0)+\lambda_{2}^{2}(0)+\lambda_{3}^{2}(0) \neq 0$, departing from $e=(\mathbf{0}, I)$ stay in the upper half space $z \geq 0$.

Proof If $W=0$ the spatial part of such a sub-Riemannian geodesic is coplanar by Theorem 8. Application of Corollary 7 and [11, Thm.7\&8] yields the result.

Corollary 11 Let $s \mapsto \gamma(s)=(x(s), y(s), z(s), R(s))$ be a sub-Riemannian geodesic in $\left(\operatorname{SE}(3), \Delta, \mathcal{G}_{1}\right)$ with $\lambda_{6}=0$ and $\lambda_{1}^{2}(0)+\lambda_{2}^{2}(0)+\lambda_{3}^{2}(0) \neq 0$, departing from $e=$ $(\mathbf{0}, I)$, s.t. its spatial projection does not have (interior) cusps. Assume it departs from a cusp and ends towards a cusp, i.e., $\lambda_{3}(0)=0=\lambda_{3}\left(s_{\max }\right)$, where $s_{\max }>0$ by definition.

Then $z(s)>0$ for all $s \in\left(0, s_{\max }\right)$, and $z(s)=0 \Leftrightarrow\left(W=0\right.$ and $s \in\left\{0, s_{\max }\right\}$ and $\mathfrak{c}<1$ ).

Proof If $W \neq 0$ and $\lambda_{3}(0)=0$, then $\left\|\underline{\lambda}^{(2)}(0)\right\|=1$ and $\left\|\underline{\lambda}^{(1)}(0)\right\|=\mathfrak{c}$, and by Theorem 6 one has

$$
z(s)=\tilde{z}(s)-\tilde{z}(0)=(m(s) \cos \tilde{\psi}(s)-1) \frac{\underline{\lambda}^{(1)}(0) \cdot \underline{\lambda}^{(2)}(0)}{\mathfrak{c}^{2}} \quad \text { for all } s \in\left[0, s_{\text {max }}\right],
$$

with $m(s)<1$ if $s<s_{\text {max }}$ and $\underline{\lambda}^{(1)}(0) \cdot \underline{\lambda}^{(2)}(0)<0$ mandatory for $s_{\text {max }}>0$ in case $\lambda_{3}(0)=$ 0 . Now $m\left(s_{\text {max }}\right)=1$ but even then due to $\tilde{\psi}(s) \neq 0$ we have $W \neq 0 \Rightarrow z\left(s_{\text {max }}\right) \neq 0$, recall (4.12).

If $W=0$ and $\lambda_{3}(0)=0$ then by Eq. 4.10 in Theorem 6, we have for this specific case,

$$
z(s)=\tilde{z}(s)-\tilde{z}(0)=\frac{\left(\underline{\lambda}^{(2)}(s)-\underline{\lambda}^{(2)}(0)\right) \cdot \underline{\lambda}^{(1)}(0)}{\mathfrak{c}^{2}}=\frac{1}{\mathfrak{c}^{2}}\left(\mathfrak{c}^{2} \sinh s-\mathfrak{c}(\cosh s-1)\right) .
$$


Now only for $\mathfrak{c}<1$, we find two nonnegative roots $s=0$ and $s=\log \frac{1+\mathfrak{c}}{1-\mathfrak{c}}=s_{\text {max }}$. The parabola corresponding to the quadratic equation arising when setting $p=e^{s}$ and multiplying with $p$ is a parabola that opens downward so that $z(s)>0$ if $s \in\left(0, s_{\max }\right)$.

Corollary 12 Let $\left(\mathbf{x}_{1},-\mathbf{e}_{z}\right)$ be the end condition of $\mathbf{P}_{\text {curve }}$ with the initial condition $\left(\mathbf{0}, \mathbf{e}_{z}\right)$. Then, a solution to problem $\mathbf{P}_{\text {curve }}$ exists if and only if $\mathbf{x}_{1} \cdot \mathbf{e}_{z}=0$. Moreover, this condition is only possible for curves departing from a cusp and ending in a cusp.

Proof Let $\mathbf{x}$ be a solution to problem $\mathbf{P}_{\text {curve with }} \mathbf{x}^{\prime}(0)=-\mathbf{x}^{\prime}(L)$ for some $0<L \leq s_{\max }$. So we have $\tilde{\mathbf{x}}^{\prime}(0)=-\tilde{\mathbf{x}}^{\prime}(L)$, which implies $\tilde{x}^{\prime}(0)=-\tilde{x}^{\prime}(L)$. But this is possible if and only if $\tilde{x}^{\prime}(0)=0=\tilde{x}^{\prime}(L)$, which is possible only if $\left\|\underline{\lambda}^{(2)}(0)\right\|=1$ and $L=s_{\text {max }}$, i.e., if the geodesic both starts and ends at a cusp. Then $z\left(s_{\max }\right)=0$ (see Corollary 11$)$.

\subsection{Symmetries of the Exponential Map}

We now describe the symmetries of the exponential map of $\mathbf{P}_{\text {curve }}$, recall Definition 6. Here, we are interested in the symmetries that retain curvature and torsion along the curve and preserve direction of time (i.e., we do not consider the symmetries involving time inversion $s \mapsto L-s$, cf. [25]). From the conservation law

$$
\left(\lambda_{1}(s)\right)^{2}+\left(\lambda_{2}(s)\right)^{2}-\left(\left(\lambda_{4}(s)\right)^{2}+\left(\lambda_{5}(s)\right)^{2}\right)=\mathfrak{c}^{2}-1
$$

and Eq. 4.17 in Theorem 7, we deduce the following corollary.

Corollary 13 Let $P \in \mathbb{R}^{6 \times 6}$ be given by

$$
P=\left(\begin{array}{cc|cc}
Q & 0 & 0 & 0 \\
0 & 1 & 0 & 0 \\
\hline 0 & 0 & \operatorname{det}(Q) Q & 0 \\
0 & 0 & 0 & 1
\end{array}\right)
$$

with $Q \in O(2)$ arbitrary. Then we have the following symmetry property of the exponential map:

$$
\widetilde{\operatorname{Exp}}_{e}\left(\lambda(0) P^{T}, l\right)=\left(\mathbf{0},\left(\begin{array}{ll}
Q & 0 \\
0 & 1
\end{array}\right)\right) \cdot \widetilde{\operatorname{Exp}_{e}}(\boldsymbol{\lambda}(0), l) \cdot\left(\mathbf{0},\left(\begin{array}{cc}
Q^{T} & 0 \\
0 & 1
\end{array}\right)\right) .
$$

Here $\lambda=\left(\lambda_{1}, \ldots, \lambda_{6}\right)$ and the group product $\cdot$ is on the Euclidean group $E(3)$.

Proof Proof can be found in [16].

Figure 6 depicts both the rotational and reflectional symmetries of the cuspless subRiemannian geodesics of problem $\mathbf{P}_{\text {mec }}$. To generate the figures, we have set

$$
\underline{\lambda}^{(2)}(0)=\left\|\underline{\lambda}^{(2)}(0)\right\|(\cos \theta, \sin \theta)^{T} \text { and } \underline{\lambda}^{(1)}(0)=\left\|\underline{\lambda}^{(1)}(0)\right\|(\cos (\theta-\Theta), \sin (\theta-\Theta))^{T} \text {. }
$$

Here, $\Theta$ denotes the angle between $\lambda^{(2)}(0)$ and $\lambda^{(1)}(0)$. For both these figures, we fixed $\left\|\underline{\lambda}^{(2)}(0)\right\|$ and $\left\|\underline{\lambda}^{(1)}(0)\right\|$. For Fig. 6a, we took $\Theta=0$ and varied $\theta$. For Fig. $6 b$, we fixed $\theta$ and varied $\Theta$. The plane of reflection corresponds to $\Theta=0$. See [16, Fig. 5] for an intuitive explanation of the relation of $\Theta$ with respect to the momentum variables. 

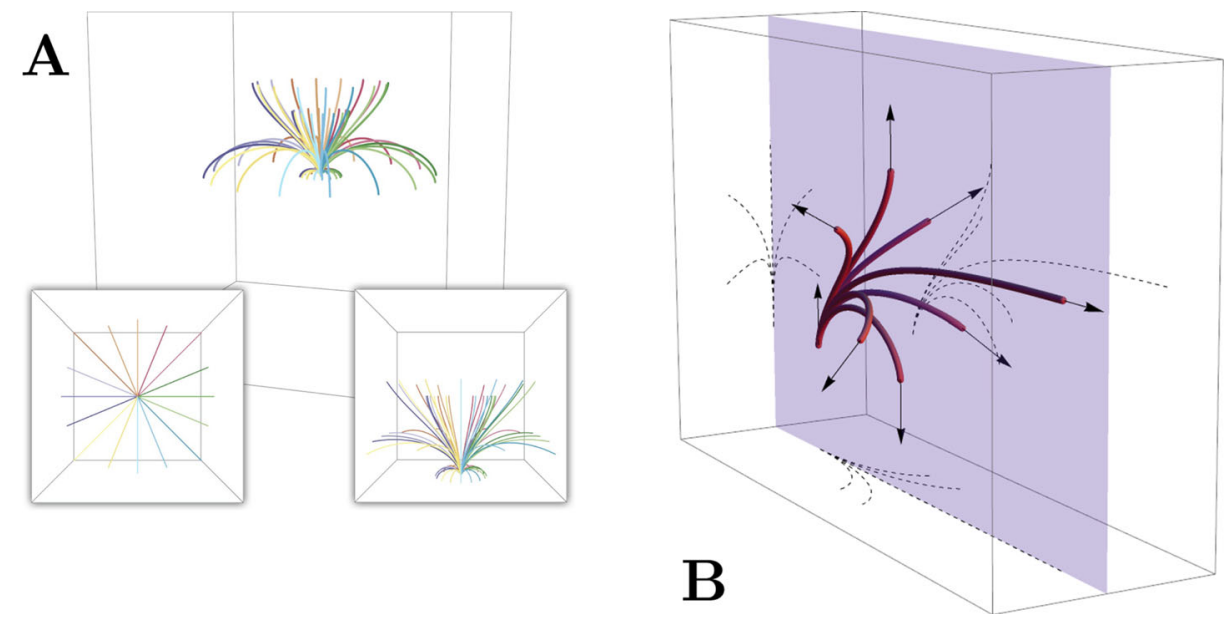

Fig. 6 a Rotational symmetries in case of several planar cuspless sub-Riemannian geodesics of problem $\mathbf{P}_{\text {mec }}$ departing from $e$ in direction of $\mathbf{e}_{z}$. b Reflectional symmetry of certain cuspless geodesics of $\mathbf{P}_{\text {mec }}$. These curves are produced by rotating $\underline{\lambda}^{(1)}(0)$ by certain angles while keeping $\underline{\lambda}^{(2)}(0)$ fixed. The plane of reflection contains the middle curve with $\underline{\lambda}^{(1)}(0)$ parallel to $\underline{\lambda}^{(2)}(0)$

\section{Numerical Analysis of Problem $P_{\text {curve }}$}

\subsection{Numerical Computations of the Jacobian of Exponential Map}

In this section, we provide a numerical investigation into the absence of conjugate points on sub-Riemannian geodesics associated to the problem $\mathbf{P}_{\text {curve }}$. Recall that a conjugate point is a critical value of the exponential map (cf. Definition 6), i.e., at such a point one has $\operatorname{det}\left(\frac{\partial(\operatorname{Exp}(\lambda(0), L))}{\partial(\lambda(0), L)}\right)=0$.

Denote by $J$ the Jacobian of the exponential map, i.e.,

$$
J=\operatorname{det}\left(\frac{\partial\left(\operatorname{Exp}\left(\lambda_{1}(0), \lambda_{2}(0), \lambda_{4}(0), \lambda_{5}(0), L\right)\right)}{\partial\left(\lambda_{1}(0), \lambda_{2}(0), \lambda_{4}(0), \lambda_{5}(0), L\right)}\right) .
$$

To compute the Jacobian numerically, we approximate the partial derivatives by finite differences:

$$
\begin{aligned}
\frac{\partial\left(\operatorname{Exp}\left(\lambda_{1}(0), \lambda_{2}(0), \ldots, L\right)\right)}{\partial\left(\lambda_{1}(0)\right)} & \approx \frac{\operatorname{Exp}\left(\lambda_{1}(0)+\Delta, \lambda_{2}(0), \ldots, L\right)-\operatorname{Exp}\left(\lambda_{1}(0)-\Delta, \lambda_{2}(0), \ldots, L\right)}{2 \Delta}, \\
\ldots & \ldots \\
\frac{\partial\left(\operatorname{Exp}\left(\lambda_{1}(0), \lambda_{2}(0), \ldots, L\right)\right)}{\partial L} & \approx \frac{\operatorname{Exp}\left(\lambda_{1}(0), \lambda_{2}(0), \ldots, L+\Delta\right)-\operatorname{Exp}\left(\lambda_{1}(0), \lambda_{2}(0), \ldots, L-\Delta\right)}{2 \Delta} .
\end{aligned}
$$

We verified that the Jacobian is always positive for a million random points within the domain $\mathcal{D}_{0}$ of the exponential map of $\mathbf{P}_{\text {curve }}$, recall (2.10). The points were determined as follows. The first integrals $\mathfrak{c}^{2}=\sum_{i=1}^{3} \lambda_{i}^{2}(0)$ and $\sum_{i=3}^{5} \lambda_{i}^{2}(0)=1$ allow us to introduce coordinates

$$
\left\{\begin{array} { l } 
{ \lambda _ { 4 } ( 0 ) = \operatorname { s i n } \phi _ { 2 } \operatorname { c o s } \phi _ { 1 } , } \\
{ \lambda _ { 5 } ( 0 ) = \operatorname { s i n } \phi _ { 2 } \operatorname { s i n } \phi _ { 1 } , } \\
{ \lambda _ { 3 } ( 0 ) = \operatorname { c o s } \phi _ { 2 } , }
\end{array} \quad \left\{\begin{array}{l}
\lambda_{1}(0)=r \cos \phi_{3}, \\
\lambda_{2}(0)=r \sin \phi_{3},
\end{array} \quad \text { where } r=\sqrt{\mathfrak{c}^{2}-\cos ^{2} \phi_{2}}=\sqrt{\mathfrak{c}^{2}-\lambda_{3}^{2}(0)} .\right.\right.
$$


By the rotational symmetry presented in Corollary 13 , we can reduce one parameter by setting $\phi_{1}=0$. Furthermore, we recall that $\lambda_{3}(0) \geq 0$, which implies $-\frac{\pi}{2} \leq \phi_{2} \leq \frac{\pi}{2}$. Thus, we can parameterize the domain of exponential map by $\phi_{2} \in\left[-\frac{\pi}{2}, \frac{\pi}{2}\right], \phi_{3} \in[0,2 \pi], \mathfrak{c} \in$ $\left[\cos \phi_{2},+\infty\right), L \in\left(0, s_{\max }\right]$. We consider $\mathfrak{c} \in\left[\cos \phi_{2}, 10\right]$, and compute the Jacobian in both a random and a uniform grid on $\left(\phi_{2}, \phi_{3}, \mathfrak{c}, L\right)$. Here, the restriction of $\mathfrak{c}$ from above is not crucial, as $s_{\max } \rightarrow 0$ when $\mathfrak{c} \rightarrow \infty$. Furthermore, the absence of conjugate points for short arcs of geodesics follows from general theory (see [1]). Finally, by Corollary 3, we have $s_{\text {max }} \leq \frac{1}{2} \log \frac{(1+\mathfrak{c})^{2}}{1+\mathfrak{c}^{2}}$, that implies $s_{\max }<0.1$ for $\mathfrak{c}>10$.

In Fig. 7, we show several trajectories of different types (U-shaped curves for $\mathfrak{c}<1$ and S-shaped curves for $\mathfrak{c}>1$ ) and corresponding plots of the Jacobian for $s \in\left[0, s_{\max }\right]$. Remarkably, the Jacobian is not just positive, it is even a monotonically increasing function of $s$ for the range of the plot. A similar behavior for the Jacobian can be seen on the closely related problem $\mathbf{P}_{\text {curve }}$ on $\mathbb{R}^{2}$ (see. [11]), where the absence of conjugate points was proved.

\subsection{The Range of the Exponential Map $P_{\text {curve }}$}

There are a number of restrictions on the possible terminal points reachable by subRiemannian geodesics of problem $\mathbf{P}_{\text {mec }}$ with cuspless spatial projection. Together, such points form the range $\mathcal{R}$ of the exponential map of $\mathbf{P}_{\text {curve }}$, recall (2.11). We present some special cases which help us to get an idea about the range of the exponential map of $\mathbf{P}_{\text {curve }}$. Recall that Corollary 12 gave us the possible terminal positions (at $z=0$ ) when the final direction is opposite to the initial direction.

Based on our numerical experiments, we pose the following conjecture which is analogous to a result in the $2 \mathrm{D}$ case of finding cuspless sub-Riemannian geodesics in $\left(\operatorname{SE}(2), \tilde{\Delta}, \tilde{G}_{1}\right)[6,11]$.

Conjecture 1 Let the range of the exponential map defined in Definition 6 be denoted by $\mathcal{R}$ and let the domain $\mathcal{D}_{0}$ of the exponential map be given by Eq. 2.10 .
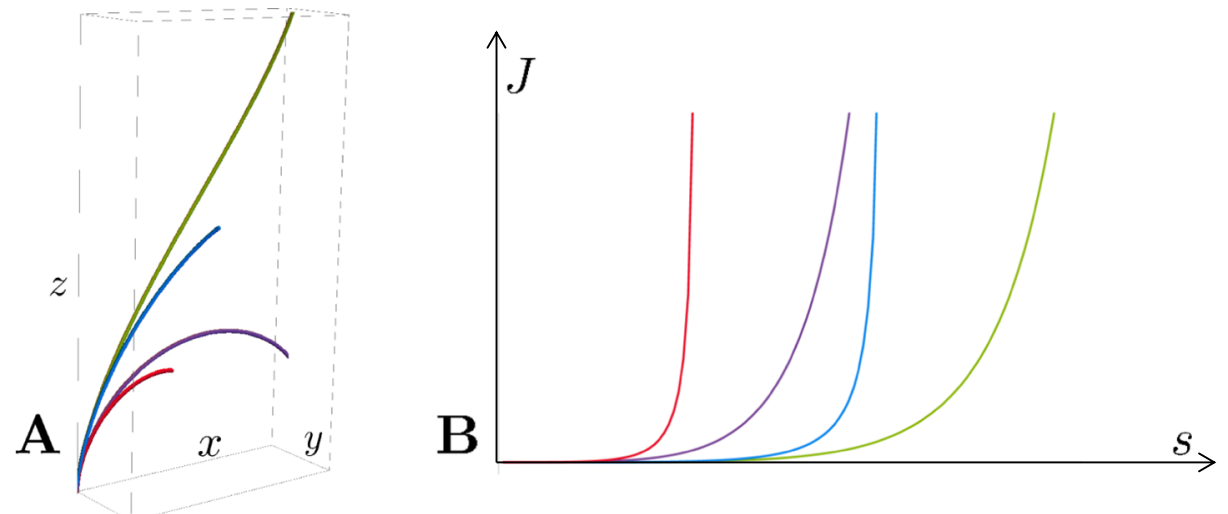

Fig. 7 a Cuspless projections of sub-Riemannian geodesics in problem $\mathbf{P}_{\mathbf{m e c}}$ of different types. U-curves are depicted in green and blue colors, and S-curves are depicted in red and purple. $\mathbf{b}$ Plot of the Jacobian of exponential map, corresponding to these geodesics. We see that the Jacobian is positive (even increasing) for all $s \in\left(0, s_{\max }\right)$. This supports our conjecture that conjugate points are absent before the first cusp point 
- $\operatorname{Exp}: \mathcal{D}_{0} \rightarrow \mathcal{R}$ is a homeomorphism when $\mathcal{D}_{0}$ and $\mathcal{R}$ are equipped with the subspace topology.

- $\operatorname{Exp}: \operatorname{int}\left(\mathcal{D}_{0}\right) \rightarrow \operatorname{int}(\mathcal{R})$ is a diffeomorphism. Here int $(S)$ denotes the interior of the set $S$.

The boundary of the range is given by $\partial \mathcal{R}=S_{B} \cup S_{R} \cup S_{L}$ with

$$
\begin{aligned}
& S_{B}=\left\{\operatorname{Exp}\left(\lambda(0), s_{\text {max }}(\lambda(0))\right) \mid \lambda(0) \in \mathcal{D}_{0}\right\} \text { and } \\
& S_{R}=\left\{\operatorname{Exp}(\lambda(0), s) \mid \lambda(0) \in \mathcal{D}_{0} \text { and } \lambda_{4}(0)^{2}+\lambda_{5}(0)^{2}=1 \text { and } s>0\right\} \\
& S_{L}=\left\{(\mathbf{0}, R) \in \operatorname{SE}(3) \mid \operatorname{Re}_{z} \cdot \mathbf{e}_{z} \geq 0\right\} .
\end{aligned}
$$
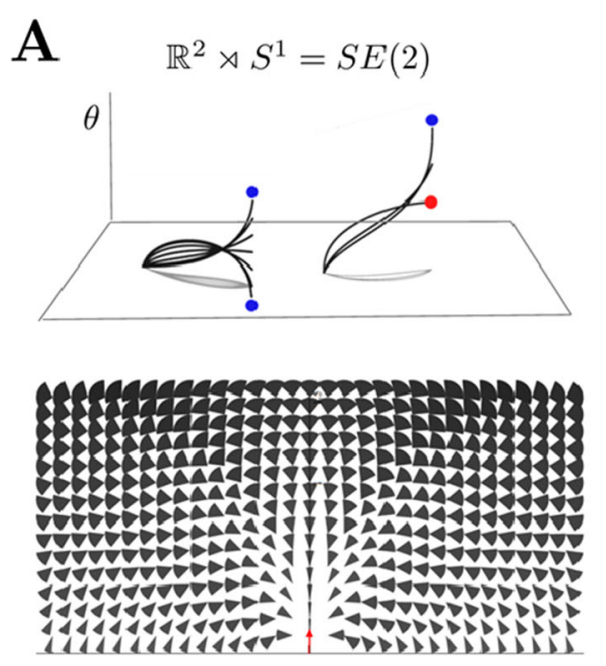

$$
\mathbb{R}^{3} \rtimes S^{2}=S E(3) /(\{0\} \times S O(2))
$$
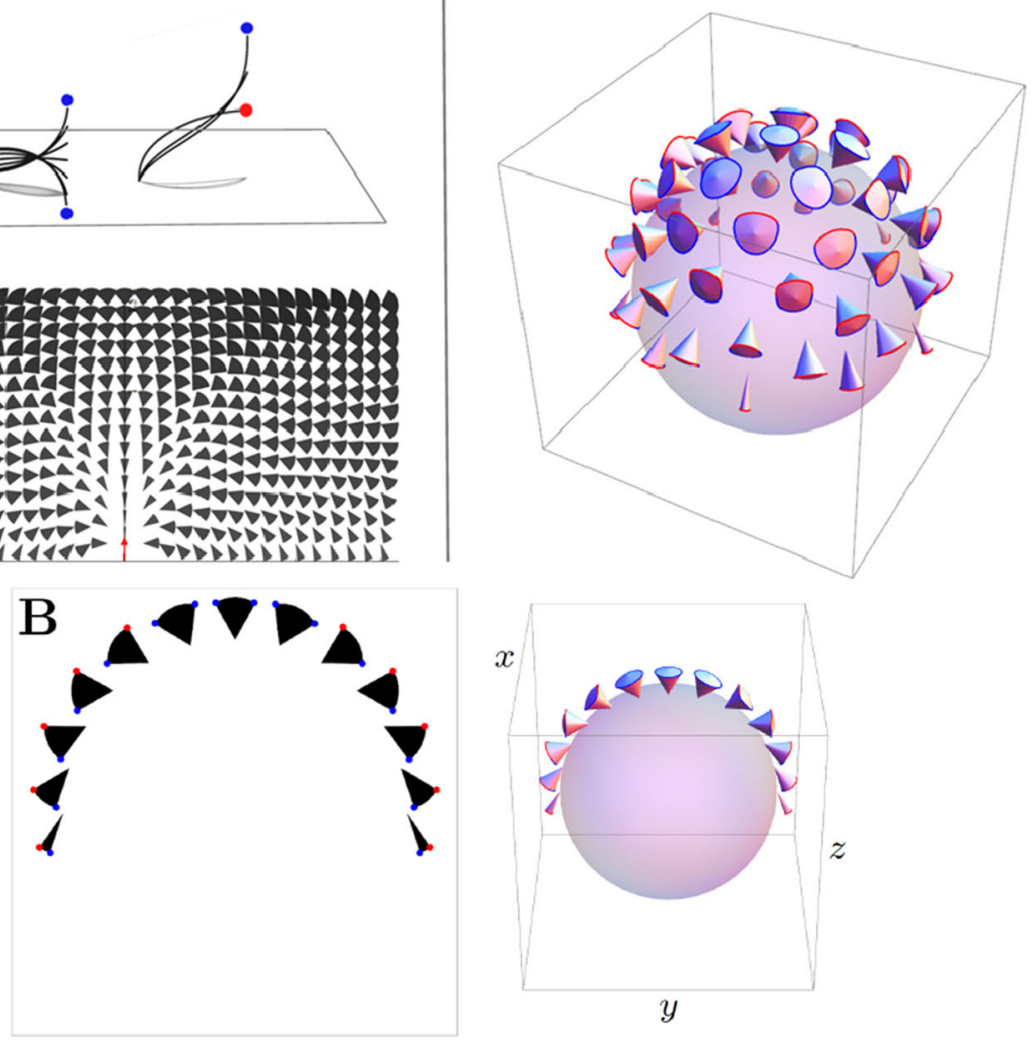

Fig. 8 a Comparison of the possible end conditions of $\mathbf{P}_{\text {curve }}$ for the $2 \mathrm{D}$ and $3 \mathrm{D}$ case. On the right, possible tangent directions of cuspless sub-Riemannian geodesics with unit length departing from the origin in the direction of $\mathbf{e}_{z}$ are depicted. In the $\operatorname{SE}(2)$ case (left) within $\left(\operatorname{SE}(2), \tilde{\Delta}, \tilde{G}_{1}\right)$ studied in [11], this set of possible directions at each point is a connected cone [11, Thm.6\&9]. The boundary is obtained by end conditions of geodesics that either begin with a cusp point (shown in red) or end at a cusp point (shown in blue). b Comparison in the special case when we set the end conditions on a half unit circle 
Note that $S_{B} \in \mathcal{R}$ and $S_{R} \in \mathcal{R}$ but $S_{L} \notin \mathcal{R}$. This conjecture would imply that no conjugate points arise within $\mathcal{R}$ and the problem $\mathbf{P}_{\text {curve }}$ (1.1) is well posed for all end conditions in $\mathcal{R}$. The proof of this conjecture would be on similar lines as in Appendix $F$ of [11]. If the conjecture is true, we have a reasonably limited set of possible directions per given end positions for which a cuspless sub-Riemannian geodesic of problem $\mathbf{P}_{\text {curve }}$ exists. Then the cones of admissible end conditions for $\mathbf{P}_{\text {curve }}$ (recall Definition 7 ) in Fig. 8a form the image of the boundary of the phase space of $\left\{\underline{\lambda}^{(2)}(0), \underline{\lambda}^{(1)}(0)\right\}$ under the exponential map. Recall Fig. 5. These cones represent the boundary of the possible reachable angles by stationary curves of problem $\mathbf{P}_{\text {curve }}$. Figure $8 \mathrm{~b}$ shows the special case of the end conditions being on a unit circle containing the $z$-axis. The final tangents are always contained within the cones at each position. Numerical computations indeed seem to confirm that this is the case (see Fig. 9). The blue points on the boundary of the cones correspond to $S_{B}$ while the red points correspond to $S_{R}$ given in Eq. 5.1.

\subsection{Solving the Boundary Value Problem Associated to $P_{\text {curve }}$}

Using explicit formulas for sub-Riemannian geodesics obtained in Theorem 6 in Section 4.2, we developed a Wolfram Mathematica package, that numerically solves the boundary value problem (BVP) associated to $\mathbf{P}_{\text {curve }}$. The package is available by link http:// bmia.bmt.tue.nl/people/RDuits/final.rar. Note, that the BVP can be also tackled by a software Hampath [10] developed to solve optimal control problems. Hampath is based on numerical integration of a Hamiltonian system of PMP and second-order optimality conditions. In contrast to Hampath, our program does not involve numerical integration, and is based on numerical solution of a system of algebraic equations in Theorem 6 , and relies on shooting based on the exact formulas, for details see [16].

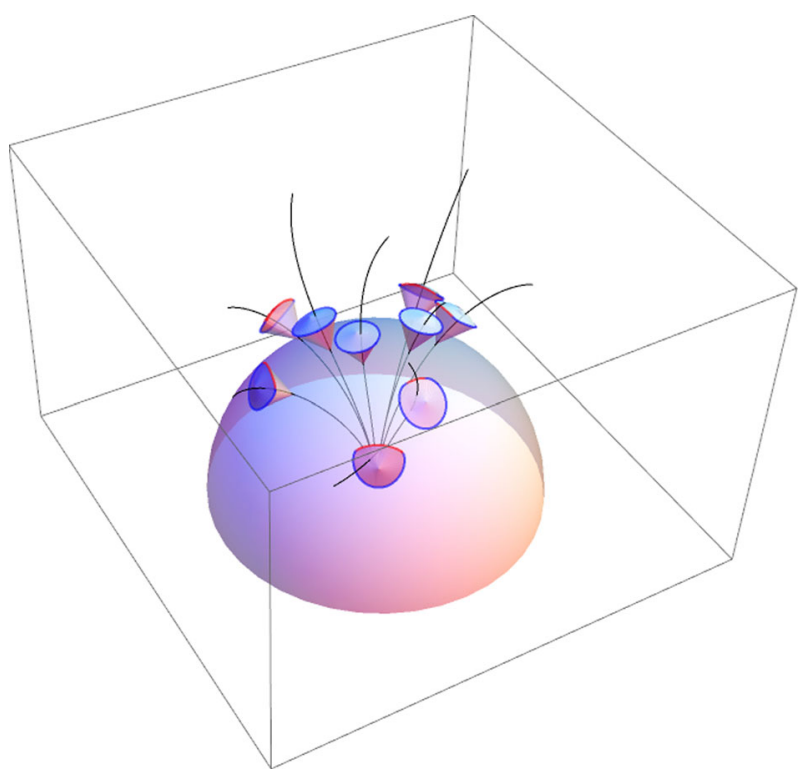

Fig. 9 The spatial part of arbitrary cuspless geodesics in $(\operatorname{SE}(3), \Delta, \mathcal{G})$ and the cones of reachable angles as depicted in Fig. 8. Note that the cuspless geodesics are always contained within the cones. We checked this for many more cases, which supports our Conjecture 1 


\section{Conclusion}

In this article, we have derived explicit exact formulas of the geodesics of problem $\mathbf{P}_{\text {curve }}$ in Theorem 6 and Corollary 4, where because of a scaling homothety we can set $\xi=1$. We have shown in Theorem 1 that they are spatial projections of special cases of subRiemannian geodesics within $\left(\operatorname{SE}(3), \Delta, \mathcal{G}_{\xi=1}\right)$ whose spatial projections are cuspless. We have characterized the set $\mathcal{R}$ of admissible end conditions for problem $\mathbf{P}_{\text {curve }}$ in Corollary 1. In Theorem 2, we have proved Liouville integrability for the corresponding sub-Riemannian problem. In Theorem 5, we have computed the first cusp time $t_{\text {cusp }}^{1}=t\left(s_{\max }\right)$ of the subRiemannian geodesics explicitly. We have shown the following geometric properties of geodesics of $\mathbf{P}_{\text {curve }}$ :

- Global bounds on torsion in Theorem 7.

- They are planar if and only if the boundary conditions are coplanar, cf. Theorem 8.

- Planar geodesics are globally optimal, cf. Corollary 7.

- They do not self intersect or roll up, cf. Corollary 8.

- They stay in the half space prescribed by the orientation of the initial condition (i.e., $z \geq$ 0 if $\mathbf{n}_{0}=\mathbf{e}_{z}$ ), which was formally shown for most cases (cf. Corollaries 9, 10, and 11). Also, we analyzed cases where the plane $z=0$ is reached, cf. Corollary 11 .

- Their rotational and reflectional symmetries in Theorem 13.

Finally, we provided a numerical Mathematica package to solve the boundary value problem via a shooting algorithm. We included numerical support for the expected absence of conjugate points on the sub-Riemannian geodesics (with $\lambda_{6}(0)=0$ ) with cuspless spatial projections, i.e., $t_{c o n j} \geq t_{c u s p}^{1}$. Also, numerical computations on the cones of reachable angles (and their boundaries, cf. Fig. 8) seem to reveal the same homeo/diffeomorphic properties of the exponential map integrating the canonical ODE's in Pontryagin maximum principle, that were formally shown on the SE(2) case [11, Thm.6\&9]. In future work, we plan to analyze the sub-Riemannian spheres via viscosity solutions of sub-Riemannian HJBequations, i.e. extend the work [3] to the SE(3) case. This will yield a numerical computation of the sub-Riemannian spheres and the 1st Maxwell-set.

\footnotetext{
Acknowledgments The research leading to these results has received funding from the European Research Council under the European Community's Seventh Framework Programme (FP7/2007-2013) / ERC grant Lie Analysis, agr. nr. 335555. The authors gratefully acknowledge EU-Marie Curie project MANET ag. no.607643 and the European Commission ITN-FIRST, agreement No. PITN-GA-2009-238702 for financial support. We gratefully acknowledge Prof. Yu.L. Sachkov for suggestions on the Liouville integrability and the co-adjoint orbit structure, and for many other suggestions on the article and its exposition. We thank Dr.ir. A.J.E.M. (Guido) Janssen for fruitful discussions on elliptic integral representation in Theorem 6, and we thank the anonymous reviewers for many valuable suggestions.
}

Open Access This article is distributed under the terms of the Creative Commons Attribution 4.0 International License (http://creativecommons.org/licenses/by/4.0/), which permits unrestricted use, distribution, and reproduction in any medium, provided you give appropriate credit to the original author(s) and the source, provide a link to the Creative Commons license, and indicate if changes were made.

\section{Appendix A: Proof of Theorem 1}

Here, we rely on the formulation of problem $\mathbf{P}_{\text {MEC }}$ using the control variables as given by Eq. 3.1 in Section 3. To this end, we note that $\left\langle\left.\omega^{i}\right|_{\gamma}, \dot{\gamma}\right\rangle=u^{i}$ for $i \in\{3,4,5\}$. So for $i=3$, 
we have $\left\langle\left.\omega^{3}\right|_{\gamma}, \dot{\gamma}\right\rangle>0 \Leftrightarrow u^{3}>0$. Particularly, this holds for a smooth minimizer $\gamma=\gamma^{*}$ of problem $\mathbf{P}_{\text {MEC. }}$.

If the end-condition $g=g_{1}=\left(\mathbf{x}_{1}, R_{1}\right)$ in Eq. 3.2 is chosen such that the optimal control $u_{3}(t)>0$ for $t \in(0, T)$, then $\frac{d s}{d t}(t)>0$ and the minimizer is parameterizable by spatial arclength $s$. Let $\gamma$ be a horizontal curve in $\left(\operatorname{SE}(3), \Delta, \mathcal{G}_{\xi}\right)$. We define $\bar{\gamma}(s):=\gamma(t(s))=$ $(\mathbf{x}(s), \mathbf{n}(s))$, and $\bar{u}^{k}(s)=u^{k}(t(s))$ and let us recall $\gamma$ is horizontal, i.e.,

$$
\dot{\gamma}(t)=\left.\sum_{i=3}^{5} u^{i}(t) \mathcal{A}_{i}\right|_{\gamma(t)}, \bar{\gamma}^{\prime}(s)=\left.1 \mathcal{A}_{3}\right|_{\bar{\gamma}(s)}+\left.\bar{u}^{4}(s) \mathcal{A}_{4}\right|_{\bar{\gamma}(s)}+\left.\bar{u}^{5}(s) \mathcal{A}_{5}\right|_{\bar{\gamma}(s)} .
$$

Lifting of a curve $\overline{\mathbf{x}}(\cdot)$ to a curve $(\mathbf{x}(\cdot), \mathbf{n}(\cdot))$ into $\mathbb{R}^{3} \rtimes S^{2}$ is done by setting $\mathbf{x}^{\prime}(s)=$ $\mathbf{n}(s)$. Let $c_{i, j}^{k}$ denote the usual structure constants of the Lie algebra of SE(3) (see Table 1), then

$$
\begin{aligned}
\mathbf{x}^{\prime \prime}(s) & =\mathbf{n}^{\prime}(s)=\frac{d}{d s} \mathbf{x}^{\prime}(s)=\left.\frac{d}{d s} \mathcal{A}_{3}\right|_{\bar{\gamma}(s)}=\left.\sum_{j, k=1}^{3} c_{j, 3}^{k}\left\langle\left.\omega^{j}\right|_{\bar{\gamma}(s)}, \bar{\gamma}^{\prime}(s)\right\rangle \mathcal{A}_{k}\right|_{\bar{\gamma}(s)} \\
& =-\left.\bar{u}^{4}(s) \mathcal{A}_{2}\right|_{\bar{\gamma}(s)}+\left.\bar{u}^{5}(s) \mathcal{A}_{1}\right|_{\bar{\gamma}(s)} .
\end{aligned}
$$

Direct computation of the Frenet-Seret ODE along horizontal curves (cf. [16, ch:2,Thm3.16]) yields the following expressions for curvature magnitude, and torsion magnitude:

$$
|\kappa(s)|^{2}=\left|\bar{u}^{4}(s)\right|^{2}+\left|\bar{u}^{5}(s)\right|^{2}, \tau(s)=\frac{\bar{u}^{4}(s)\left(\bar{u}^{5}\right)^{\prime}(s)-\bar{u}^{5}(s)\left(\bar{u}^{4}\right)^{\prime}(s)}{\left|\bar{u}^{4}(s)\right|^{2}+\left|\bar{u}^{5}(s)\right|^{2}} .
$$

Furthermore, we have $\|\left(\mathbf{x}^{\prime}(s) \|=\bar{u}^{3}(s)=1\right.$, and we see that the energy functionals of $\mathbf{P}_{\text {curve }}$ and $\mathbf{P}_{\text {mec }}$ coincide, as we have

$$
\int_{0}^{T} \sqrt{\left.\mathcal{G}_{\xi}\right|_{\gamma(t)}(\dot{\gamma}(t), \dot{\gamma}(t))} \mathrm{d} t=\int_{0}^{L} \sqrt{\left.\mathcal{G}_{\xi}\right|_{\bar{\gamma}(s)}\left(\bar{\gamma}^{\prime}(s), \bar{\gamma}^{\prime}(s)\right)} \mathrm{d} s=\int_{0}^{L} \sqrt{\kappa^{2}(s)+\xi^{2}} \mathrm{~d} s .
$$


horizontal part

$$
\dot{\gamma}=\left.\lambda^{3} \mathcal{A}_{3}\right|_{\gamma}+\left.\lambda^{4} \mathcal{A}_{4}\right|_{\gamma}+\left.\lambda^{5} \mathcal{A}_{5}\right|_{\gamma},
$$

and for the vertical part, we obtain the ODE

$$
\begin{aligned}
& \frac{\mathrm{d}}{\mathrm{d} t} \lambda_{i}=-\sum_{b=3}^{5} \sum_{l=1}^{6} c_{i, b}^{l} \lambda^{b} \lambda_{l} \Leftrightarrow \\
& \frac{\mathrm{d}}{\mathrm{d} t}\left(\lambda_{1}, \lambda_{2}, \lambda_{3}, \lambda_{4}, \lambda_{5}, \lambda_{6}\right)=\left(\lambda_{3} \lambda_{5}, \lambda_{3} \lambda_{4}, \lambda_{1} \lambda_{5}-\lambda_{2} \lambda_{4}, \xi^{-2} \lambda_{3} \lambda_{2}-\lambda_{5} \lambda_{6},-\xi^{-2} \lambda_{3} \lambda_{1}+\lambda_{4} \lambda_{6}, 0\right) .
\end{aligned}
$$

Note that reciprocal momentum components are related by the inverse metric tensor and thereby given by $\lambda^{3}=\xi^{-2} \lambda_{3}, \lambda^{4}=\lambda_{4}, \lambda^{5}=\lambda_{5}$. PMP gives us that the stationary curves obtained via these ODE's are short time local minimizers. It also provides us the Hamiltonian $H(\lambda)=\frac{1}{2}\left(\xi^{-2} \lambda_{3}^{2}+\lambda_{4}^{2}+\lambda_{5}^{2}\right)$ and the Exponential map.

Now, we must choose $\gamma(L)=g_{1} \in \mathrm{SE}(3)$ from the equivalence class $\left[g_{1}\right]=\{g \in$ $\left.\mathrm{SE}(3) \mid g \sim g_{1}\right\}$ (i.e., left-coset recall (2.5)) such that the minimum in Eq. 2.5 is attained. This does not hold for all elements in $\operatorname{SE}(3)$. In fact, it only holds for those end conditions that can be reached with geodesics having $\lambda_{6}(0)=0$. This follows from the fact that along all sub-Riemannian geodesics one has $\dot{\lambda}_{6}=0$ and the fact that the sub-Riemannian minimizers with $\lambda_{6}=0$ are precisely the ones where the constraint $\omega^{6}=0$ is redundant and the result follows. See also Fig. 4. 


\section{Appendix B: Cartan Connection $\bar{\nabla}$ on Sub-Riemannian Manifold $\left(\mathrm{SE}(3), \Delta, \mathcal{G}_{\xi}\right)$}

In this appendix, we provide background/embedding of Definition 9 and Theorem 3, and in particular Eqs. 3.7 and 3.8, into theory of Cartan connections.

The - Cartan connection [8] is induced by the Maurer-Cartan form $\left(L_{g^{-1}}\right)_{*}$ which induces a Cartan-Ehresmann connection on the principal G-bundle $P=(\operatorname{SE}(3),\{e\}, \pi, R)$, with total space $\mathrm{SE}(3)$, base space $\{e\}=\operatorname{SE}(3) / \mathrm{SE}(3)$, projection $\pi(g)=e$, and the right action $R_{g_{1}} g_{2}=g_{2} g_{1}$.

The construction is as follows. The Maurer-Cartan form induces a connection $\tilde{\omega}$ on the associated vector bundle $\operatorname{SE}(3) \times{ }_{A d} \mathcal{L}(\operatorname{SE}(3))$, where $\mathcal{L}(\mathrm{SE}(3))$ denotes the Lie algebra of left-invariant vector fields, given by $\tilde{\omega}=\sum_{j=1}^{6}(A d)_{*} \mathcal{A}_{j} \otimes \omega^{j}=\sum_{i, j, k=1}^{6} c_{i, j}^{k} \mathcal{A}_{k} \otimes$ $\omega^{i} \otimes \omega^{j}$. The form $\tilde{\omega}$ induces a matrix-valued 1 form $-\tilde{\omega}\left(\omega^{k}, \cdot, \mathcal{A}_{j}\right)$ on the frame bundle, and moreover it induces a connection $\nabla$ on tangent bundle $T(\operatorname{SE}(3))$, where $\nabla_{\left(\sum_{i=1}^{6} \dot{\gamma}^{i} \mathcal{A}_{i}\right)}\left(\sum_{k=1}^{6} a^{k} \mathcal{A}_{k}\right)=\sum_{k=1}^{6}\left(\dot{a}^{k}-\sum_{i, j=1}^{6} c_{i, j}^{k} \dot{\gamma}^{i} a^{j}\right) \mathcal{A}_{k}$. This is all still in the Riemannian setting.

In the sub-Riemannian setting of $\left(\operatorname{SE}(3), \Delta, \mathcal{G}_{\xi}\right)$, one relies on a different structure subgroup $\widetilde{\mathrm{SE}(2)}$ (consisting of translations and rotations in the $x y$-plane only) isomorphic to $\mathrm{SE}(2)$, rather than structure group SE(3) in the Riemannian setting. This boils down to constraining some of the summation indices and therefore we use $\bar{\nabla}$ given by Eq. 3.7 instead of $\nabla$. Next, we explain how partial connection $\bar{\nabla}$ appears in Cartan geometry.

In the sub-Riemannian manifold $\left(\operatorname{SE}(3), \Delta, \mathcal{G}_{\xi}\right)$, with $\Delta=\operatorname{ker}\left\{\omega^{1}\right\} \bigcap \operatorname{ker}\left\{\omega^{2}\right\} \bigcap \operatorname{ker}\left\{\omega^{6}\right\}$, the directions $\mathcal{A}_{1}, \mathcal{A}_{2}$, and $\mathcal{A}_{6}$ are prohibited. To get a better grasp on what this means on the manifold level, we consider principal fibre bundles. To this end, we consider the subgroup isomorphic to $\mathrm{SE}(2)$ given by $\widehat{\operatorname{SE}(2)}=\left\{\exp \left(c^{1} A_{1}+c^{2} A_{2}+c^{6} A_{6}\right) \mid c^{1}, c^{2}, c^{6} \in \mathbb{R}\right\}$ with $A_{k}=\left.\mathcal{A}_{k}\right|_{e}$.

Now, we consider the principal fibre bundle $\bar{P}=(\mathrm{SE}(3), \operatorname{SE}(3) / \widetilde{\operatorname{SE}(2)}, \pi, R)$ with $R_{h} g=g h, h \in \widetilde{\mathrm{SE}(2)}, \pi(g)=[g]=g \widehat{\mathrm{SE}(2)} \in \mathrm{SE}(3) / \widetilde{\mathrm{SE}(2)}$. On $\bar{P}$, we consider the Maurer-Cartan form $\bar{w}=\left(L_{h^{-1}}\right)_{*}$, more precisely $\bar{w}\left(\mathcal{A}_{g}\right)=\sum_{i=3}^{5}\left\langle\left.\omega^{i}\right|_{g}, X_{g}\right\rangle \mathcal{A}_{i}$.

Via the group representation $\widehat{\mathrm{SE}(2)} \ni h \mapsto A d(h):=\left(L_{h^{-1}} R_{h}\right)_{*}$, we obtain the associated vector bundle (def. 3.7 in [13]) $\left(\mathrm{SE}(3) \times_{A d \mid \widetilde{\mathrm{SE}(2)}} \mathcal{L}(\mathrm{SE}(3))\right)$ with corresponding connection form

$$
\bar{w}=\sum_{j=3}^{5}(A d \mid \widetilde{S E(2)})_{*}\left(\mathcal{A}_{j}\right) \otimes \omega^{j}=\sum_{j=3}^{5} a d\left(\mathcal{A}_{j}\right) \otimes \omega^{j}=\sum_{i, j, k=3}^{5} c_{i, j}^{k} \mathcal{A}_{k} \otimes \omega^{i} \otimes \omega^{j},
$$

where $\operatorname{ad}(X)=[\cdot, X]$. This yields a $3 \times 3$ matrix valued one form on the frame bundle $\bar{w}_{j}^{k}=$ $-\bar{w}\left(\omega^{k}, \cdot, \mathcal{A}_{j}\right)$ which yields a partial connection on the horizontal part $\Delta$ of $T(S E(3))$ :

$$
\bar{\nabla}_{X} \mathcal{A}=\sum_{k=3}^{5}\left(\dot{a}^{k} \mathcal{A}_{k}+\sum_{j=3}^{5} a^{j} \bar{w}_{j}^{k}(X) \mathcal{A}_{k}\right)=\sum_{k=3}^{5}\left(\dot{a}^{k} \mathcal{A}_{k}+\sum_{i, j=3}^{5} a^{j} \dot{\gamma}^{i} c_{j, i}^{k} \mathcal{A}_{k}\right),
$$

with $X=\sum_{i=3}^{5} \dot{\gamma}^{i} \mathcal{A}_{i}, \mathcal{A}=\sum_{k=3}^{5} a^{k} \mathcal{A}_{k}$ and $\bar{w}_{j}^{k}\left(\mathcal{A}_{i}\right)=-\bar{w}\left(\omega^{k}, \mathcal{A}_{i}, \mathcal{A}_{j}\right)=-c_{i, j}^{k}$ where the Christoffels are equal to minus the structure constants of the Lie algebra and where $\dot{a}^{k}=\sum_{i=3}^{5} \dot{\gamma}^{i}\left(\left.\mathcal{A}_{i}\right|_{\gamma} a^{k}\right)$. 
Finally, Eq. B.1 is equivalent to Eq. 3.7, as $c_{i, j}^{k}=-c_{j, i}^{k}$. As shown in the proof of Theorem 3, partial connection $\bar{\nabla}$ on the tangent bundle induces a partial connection $\bar{\nabla}^{*}$ on the cotangent bundle (given by the left-hand side of Eq. 3.8).

\section{References}

1. Agrachev AA, Sachkov YL. Control theory from the geometric viewpoint: Springer; 2004.

2. Arnold VI, Kozlov VV, Neishtadt AI. Mathematical aspects of classical and celestial mechanics. Berlin: Springer; 1997.

3. Bekkers EJ, Duits R, Mashtakov A, Sanguinetti GR, (joint main authors). A PDE approach to Datadriven Sub-Riemannian Geodesics in SE(3). SIAM J Imag Sci. 2015;8:4:2740-2770.

4. Ben-Yosef G, Ben-Shahar O. A tangent bundle theory for visual curve completion. PAMI. 2012;(34) 7:1263-1280.

5. Boscain U, Charlot G, Rossi F. Existence of planar curves minimizing length and curvature. Proceedings of the Steklov institute of mathematics; 2010.

6. Boscain U, Duits R, Rossi F, Sachkov Y. Curve cuspless reconstruction via sub-Riemannian geometry. ESAIM-COCV, Control Optimization and Calculus of Variations. 2014;20:748-770.

7. Bryant R, Griffiths P. Reduction for constrained variational problems and $(1 / 2) \kappa^{2} \mathrm{ds}$. Am J Math 1986;108(3):525-570.

8. Cartan E, Schouten J. On the geometry of the group-manifold of simple and semi-simple groups. Proc Akad Wekensch. 1926;29:803-815.

9. Citti G, Sarti A. A cortical based model of perceptional completion in the roto-translation space. J Math Imaging Vision. 2006;24(3):307-326.

10. Caillau J.-B., Cots O, Gergaud J. Differential pathfollowing for regular optimal control problems. Optim Methods Softw. 2012;27(2):177-196.

11. Duits R, Boscain U, Rossi F, Sachkov Y. Association felds via cuspless sub-Riemannian geodesics in SE(2). J Math Imaging Vision. 2014;49(2):384-417.

12. Duits R, Dela Haije TCJ, Creusen EJ, Ghosh A. Morphological and linear scale spaces for fiber enhancement in DW-MRI. JMIV. 2013;46(3):326-368.

13. Duits R, Franken EM. Left-invariant parabolic evolution equations on SE(2) and contour enhancement via invertible orientation scores, part II: nonlinear left-invariant diffusion equations on invertible orientation scores. QAM-AMS. 2010;68(2):293-331.

14. Franken E, Duits R. Crossing-preserving coherence-enhancing diffusion on invertible orienattion scores. IJCV. 2009;85(3):253-278.

15. Duits R, Franken EM. Left-invariant diffusions on the space of positions and orientations and their application to crossing-preserving smoothing of HARDI images. IJCV. 2011;92:231264.

16. Ghosh A, Duits R, Dela Haije TCJ. On sub-Riemannian geodesics within $\mathrm{SE}(3) /(\{0\} \operatorname{SO}(2))$ whose spatial projections do not have cusps. 2014. (version 4). arXiv:1305.6061v4.pdf.

17. Dela Haije TCJ, Duits R, Tax C. Sharpening fibers in diffusion weighted MRI via erosion. Visualization and processing of tensors and higher order descriptors for multi-valued data. Mathematics and visualization; 2014. p. 97-126.

18. Jurdjevic V. Geometric control theory: Cambridge University Press; 1997.

19. Kirillov AA. Lectures on the orbit method. AMS. 2004.

20. Laurent-Gengoux C, Pichereau A, Vanhaecke P. Poisson structures Grundlehren der mathematischen Wissenschaften 347. Berlin: Springer; 2013.

21. Marsden JE, Ratiu TS. Introduction to mechanics and symmetry: a basic exposition of classical mechanical systems 2nd edition (texts in applied mathematics). 1999.

22. Hladky RK, Pauls SD. Minimal surfaces in the Roto-Translation group with applications to a NeuroBiological image completion model. J Math Imaging Vis. 2010;36:1-27.

23. Mashtakov A, Ardentov A, Sachkov Y. Parallel algorithm and software for image inpainting via SubRiemannian minimizers on the group of rototranslations. Numerical Methods: Theory and Applications. 2013;6(1):95-115.

24. Mashtakov AP, Sachkov YL. Superintegrability of left-invariant sub-Riemannian structures on unimodular three-dimensional Lie groups. Differential Equations. 2015;51(11):1476-1483. 
25. Moiseev I, Sachkov YL. Maxwell strata in sub-Riemannian problem on the group of motions of a plane. ESAIM: COCV. 2010;16(2):380-399.

26. Montgomery R. A tour of subriemannian geometries, their geodesics and applications. AMS. 2002.

27. Pennec X. Bi-invariant means on Lie groups with Cartan-Schouten connections. Geometric science of information, lecture notes in computer science. Berlin: Springer; 2013. p. 59-67.

28. Petitot J. Vers une Neurogéométrie. Fibrations corticales, structures de contact et contours subjectifs modaux. Math Inform Sci Humaines. 1999;145:5-101.

29. Petitot J. Neurogéométrie de la vision - Modèles mathématiques et physiques des architectures fonctionnelles. Les Éditions de l'École Polytechnique. 2008.

30. Pontryagin LS, Boltyanskii VG, Gamkrelidze RV, Mishchenko E. The mathematical theory of optimal processes. English translation. Interscience. ISBN 2-88124-077-1. 1962.

31. Portegies JM, Fick RHJ, Sanguinetti GR, Meesters SPL, Girad G, Duits R. Improving fiber alignment in HARDI by combining contextual PDE flow with constrained spherical deconvolution. PLos ONE. 2015;10(10).

32. Sachkov Y. Conjugate and cut time in the sub-Riemannian problem on the group of motions of a plane. ESAIM: COCV. 2010;16(4):1018-1039.

33. Sachkov Y. Cut locus and optimal synthesis in the sub-Riemannian problem on the group of motions of a plane. ESAIM: COCV. 2011;17(2):293-321.

34. Sanguinetti G, Citti G, Sarti A. Image completion using a diffusion driven mean curvature flow in a subRiemannian space. International conference on computer vision theory and applications (VISAPP'08), FUNCHAL; 2008.

35. Spivak M. Differential geometry, Part 2. Berkeley: Publish or Perish; 1975.

36. Vinter R. Optimal control: Birkhauser; 2010.

37. Wilcox RM. Exponential operators and parameter differentiation in quantum physiscs. J Math Phys. 1967;8:962-982. 